\title{
A Model for interstitial drainage through a Sliding Lymphatic Valve
}

\author{
Charles Heppell, Tiina Roose $\dagger$ Giles Richardson*
}

March 17, 2015

\begin{abstract}
This study investigates fluid flow and elastic deformation in tissues that are drained by the primary lymphatic system. A model is formulated based on the Rossi hypothesis that states that the primary lymphatic valves, which are formed by overlapping endothelial cells around the circumferential lining of lymphatic capillaries, open in response to swelling of the surrounding tissue. Tissue deformation and interstitial fluid flow through the tissue are treated using the Biot equations of poroelasticity and, the fluid flux, (into the interstitium) across the walls of the blood capillaries, is assumed to be linearly related to the pressure difference across the walls via a constant of proportionality (the vascular permeability). The resulting model is solved in a periodic domain containing one blood capillary and one lymphatic capillary starting from a configuration in which the tissue is undeformed. On imposition of a constant pressure difference between blood and lymphatic capillaries the solutions are found to settle to a steady state. Given that the magnitude of pressure fluctuations in the lymphatic system is much smaller than this pressure difference between blood and lymph it is postulated that the resulting steady state solution gives a good representation of the state of the tissue under physiological conditions. The effects of changes to the Young's modulus of the tissue, the blood-lymphatic pressure difference, vascular permeability and valve dimensions on the steady state are investigated and discussed in terms of their effects on oedema in the context of age- and pregnancy-related changes to the body.
\end{abstract}

\section{Keywords}

Lymphatic system, primary lymphatic valves, Darcy's law, poroelasticity

\footnotetext{
* School of Mathematics, University of Southampton, Southampton SO17 1BJ, UK.

$\dagger$ (corresponding author) Bioengineering Sciences Research Group, Faculty of Engineering and Environment, University of Southampton, Southampton SO17 1BJ, UK.
} 


\section{Introduction}

Every day 2-4 liters of fluid is pushed into the interstitium by the pressure difference between the interior blood capillaries and their surroundings [34]. Excess interstitial fluid is drained by the lymphatic system and its failure, or partial failure, results in oedema (an abnormal accumulation of fluid in the interstitium). Interstitial fluid drainage into the lymphatic system is first mediated by the primary lymphatic valves, which are formed by overlapping endothelial cells in the lymphatic capillary wall (see figure 1). The operation of these valves is the main focus of this work. The usual hypothesis for primary lymphatic valve function is that valve opening occurs in response to pressure differences between the interstitium and the lymphatic lumen which, when they are sufficiently large, lead to the valve flaps bending into the lymphatic lumen and creating a narrow gap through which interstitial fluid can flow into the lymphatic capillary. Models based on this hypothesis include [20,38]. However here we will be primarily concerned with an alternative hypothesis, due to Rossi [46], which proposes instead that the overlapping primary lymphatic valves slide open in response to expansion of the intersititium about the lymphatic capillary, as fluid accumulates in the tissue. Some support for this hypothesis can be gained from anatomical observations that show attachment filaments between the lymphatic capillary wall and the surrounding interstitium [32]; these are presumed to increase lumenal volume (of the lymphatic capillary) as the surrounding interstitium swells [42, 52].

In this work we formulate a mathematical model based on Rossi's 'sliding door' hypothesis of lymphatic valve opening and compare our results to previous models based on pressure operated valves [20,38]. This model makes use of a poroelastic description of the interstitium and assumes that the endothelial cells in the walls of the lymphatic capillary are firmly anchored to the surrounding interstitium, except in the overlap regions that form the primary lymphatic valves. This leads to a coupling between the interstitial tissue deformation and the overlap between adjacent endothelial cells. Thus sufficiently large tissue expansion, in the immediate vicinity of the lymphatic capillary, gives rise to a gap (i.e. a negative overlap) between endothelial cells through which fluid can drain from the interstitium into the lymphatic lumen (a process which is illustrated in figure 1).

Our results show that a primary lymphatic valve, with a mechanism that obeys Rossi's hypothesis, settles to a steady state in which tissue swelling is controlled to within tight limits even when factors, such as vascular permeability or the interstitial Young's modulus, are allowed to change. This is in contrast to what would be observed if the valve mechanism were such that it opens in response to the pressure difference between the lymphatic lumen and the surrounding interstitium. From an evolutionary point of view, it would seem to us, that a primary lymphatic valve with the Rossi structure is adaptively advantageous because it serves to keep the fluid content in the interstitium fairly constant even when properties of the tissue change. 


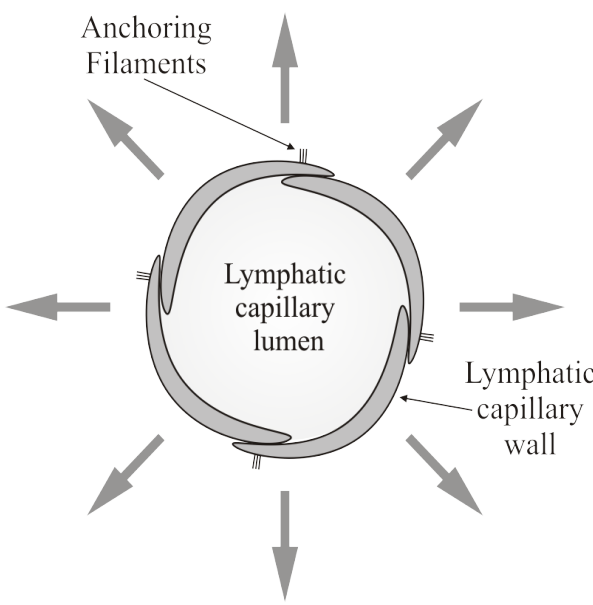

(a)

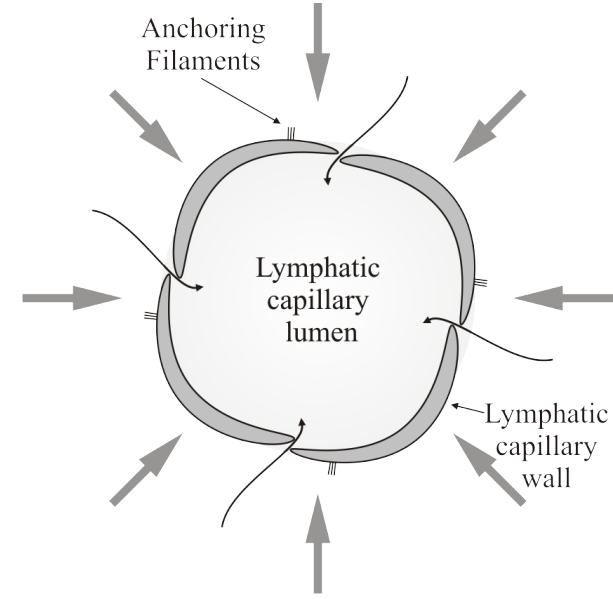

(b)

Figure 1: Two cross sectional drawings of a lymphatic capillary. The lymphatic valve cycles from closed (a) to open (b) by the expansion of the surrounding interstitium. The thick arrows in (a) and (b) indicate the direction of the tissue deformation, while the thin arrows in (b) indicate fluid motion.

In the following section we define an idealized tissue containing a periodic array of blood and lymphatic capillaries separated by the interstitium. We describe the fluid flow from the blood capillary (through the interstitium) to the lymphatic capillary, and the tissue deformation using the theory of poroelasticity. We model the valve as a sliding door that opens in response to tissue deformations. In Section 3, we numerically solve the model, using a finite element package, and present our results. The implementation of our numerical simulation is discussed in appendix A. Finally in section 4 we present our conclusions.

\section{Model Development}

Here we develop a model for lymphatic drainage of a tissue comprising a periodic array of blood and lymphatic capillaries (as illustrated in Figure 2). We treat the interstitial space lying between the blood and lymphatic capillaries as a linear poroelastic medium. Blood enters the interstitium from blood capillaries in response to the pressure difference between the blood and the interstitial fluid (the resistance of the blood capillary membrane to this flow is considered to be linearly proportional to the flow rate). Drainage of fluid from the interstitium takes place through lymphatic valves in the wall of the lymphatic capillary that open in response to the local deformation of the interstitium (i.e. they only open once the circumference of the lymphatic capillary has exceeded a critical size).

The model geometry is designed to be consistent with the literature. Measurements by Leak [31] found the radii of lymphatic capillaries to be of the order of $10 \times 10^{-6} \mathrm{~m}$, i.e. slightly larger than an average blood capillary [7]. Measurements by Geleff et al and Sauter et al [19, 48] found distances between blood and lymphatic capillaries of the order of $10^{-4} \mathrm{~m}$. Lymphatic capillaries are known to follow 
blood capillaries in close proximity around the entire body [8,30]. Given the lack of accurate three-dimensional data on real vessel geometries we opt to solve our problem over an idealized periodic geometry, displayed in Figure 2 (arrows indicate the fluid movement), in which blood and lymphatic capillaries are distributed uniformly, in a square lattice, throughout the tissue. Given that lymph and blood capillaries are distributed more or less uniformly throughout a particular tissue we expect this assumption to lead to only small quantitative discrepancies between the results obtained on our idealised geometry and a realistic three-dimensional geometry. The choice of such a periodic array allows us to characterize the behavior of the entire tissue by a single periodic tile (represented by the dotted square in Figure 2). This tile contains the fluid flow from one blood capillary to one lymphatic capillary.

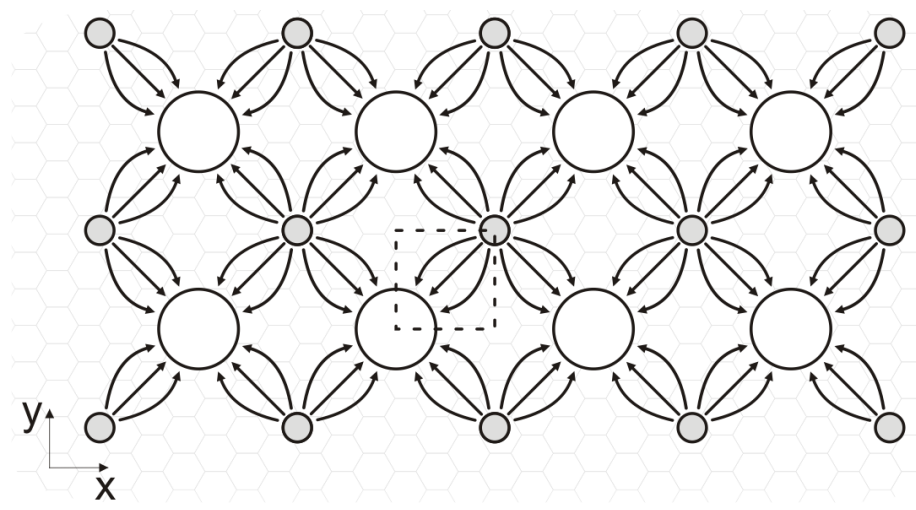

Figure 2: A cross sectional view of an idealized tissue with blood (grey) and lymphatic (white) capillaries surrounded by the interstitium. The arrows indicate the fluid movement.

\section{Valve Mechanics}

The primary lymphatic valve system controls the rate at which interstitial fluid drains into the lymphatics. We model this valve as a 'sliding door' that opens and closes in response to the expansions and contractions of the surrounding interstitium, respectively. That is, we take the valve to be closed if its circumference, $C(t)$, is less than or equal to a critical value $C_{c r i t}$, and open if it exceeds this value. Thus,

$$
\begin{aligned}
& \text { if } C(t)<C_{\text {crit }} \text { then valve closed, } \\
& \text { if } C(t)>C_{\text {crit }} \text { then valve open. }
\end{aligned}
$$

\subsection{Governing Equations}

Here we model the coupled interstitial fluid flow and deformations by Biot's equations of poroelasticity [5] (these describe the flow of a Newtonian fluid through a deformable linear elastic porous medium). This approach has been adopted 
previously in biological tissues, and validated against experiements, in $[22,54]$. A comprehensive review of the theory is provided in [6].

\subsubsection{The Fluid Equations}

In the Biot model, the fluid flux relative to the solid $\left(\mathbf{Q}_{f}\right)$ is related to the fluid pore pressure gradient $(\nabla P)$ by Darcy's law,

$$
\mathbf{Q}_{f}=-K \nabla P
$$

where $K\left(\mathrm{~m}^{3} \mathrm{skg}^{-1}\right)$ is the interstitial hydraulic conductivity. Here we assume that $K$ is constant (i.e. independent of tissue deformation), an assumption that is only valid when tissue deformations remain small. We can write the fluid flux $\mathbf{Q}_{f}$ in terms of the difference in velocities of the fluid $\left(\mathbf{v}^{f}\right)$ and solid $\left(\boldsymbol{v}^{s} \approx \partial \mathbf{u} / \partial t\right)$ by,

$$
\mathbf{Q}_{f}=\phi\left(\mathbf{v}^{f}-\frac{\partial \mathbf{u}}{\partial t}\right)
$$

where $\phi$ is the fluid volume fraction.

Both the interstitial fluid and the solid material making up the matrix are assumed to be separately incompressible. The assumption that the solid matrix is incompressible does not however imply that the interstitium as a whole is incompressible since it can be compressed by allowing fluid to flow out of its pores. Conservation of fluid and solid volumes gives,

$$
\begin{aligned}
\frac{\partial \phi}{\partial t}+\nabla \cdot\left(\phi \mathbf{v}^{f}\right) & =0 \\
-\frac{\partial \phi}{\partial t}+\nabla \cdot\left((1-\phi) \frac{\partial \mathbf{u}}{\partial t}\right) & =0 .
\end{aligned}
$$

The addition of (2.4) and (2.5) results in the relation,

$$
\nabla \cdot\left[\phi\left(\mathbf{v}^{f}-\frac{\partial \mathbf{u}}{\partial t}\right)\right]=-\nabla \cdot\left(\frac{\partial \mathbf{u}}{\partial t}\right) .
$$

On substituting the term $\phi\left(\mathbf{v}^{f}-\partial \mathbf{u} / \partial t\right)$ for $-K \nabla P$, using equations (2.2)-(2.3), in the above we obtain the following equation for conservation of fluid:

$$
\nabla \cdot(K \nabla P)=\nabla \cdot\left(\frac{\partial \mathbf{u}}{\partial t}\right) .
$$

Furthermore, since the geometry we consider is 2 -d, we need only consider planestrain and pressure variation within the plane, so that

$$
\mathbf{u}=\left(u_{1}(x, y, t), u_{2}(x, y, t), 0\right) \quad \text { and } \quad P=P(x, y, t),
$$




\subsubsection{Solid Governing Equations}

In Biot's model, the elastic stress strain relation is linear since only small deformations are considered, but crucially depends upon the fluid pore pressure [5]. It thus takes the form,

$$
\begin{aligned}
\sigma_{i j} & =2 \mu \epsilon_{i j}+\lambda \delta_{i j} \epsilon_{k k}-P \delta_{i j} \\
\epsilon_{i j} & =\epsilon_{j i}=\frac{1}{2}\left(\frac{\partial u_{i}}{\partial x_{j}}+\frac{\partial u_{j}}{\partial x_{i}}\right),
\end{aligned}
$$

where $\sigma_{i j}$ is the stress tensor, $\epsilon_{i j}$ is the strain tensor, $u_{i}$ is the tissue displacement, $\delta_{i j}$ is the Kronecker delta tensor, and $\lambda(\mathrm{Pa})$ and $\mu(\mathrm{Pa})$ are the first and second drained Lamé parameters.

These drained Lamé parameters are calculated by subjecting a material volume of the porous medium to an external load and measuring the resulting strains while allowing the fluid contained within the volume to freely drain out of it (or enter it) at constant fluid pressure $P$. Generally parameters in problems where the movement of fluid and solid needs to be calculated independently are calculated under drained conditions.

Neglecting inertial forces and any external body forces in the solid leads to a force balance equation of the form,

$$
\frac{\partial \sigma_{i j}}{\partial x_{j}}=0 .
$$

Substituting the stress strain relationship (2.9) into (2.11) under the assumption of plane strain, (2.8), yields two equations for the displacements $u_{1}$ and $u_{2}$ in terms of pressure gradient, i.e.,

$$
\begin{aligned}
& (2 \mu+\lambda) \frac{\partial^{2} u_{1}}{\partial x_{1}^{2}}+\lambda \frac{\partial^{2} u_{2}}{\partial x_{1} \partial x_{2}}-\frac{\partial P}{\partial x_{1}}+\mu\left(\frac{\partial^{2} u_{1}}{\partial x_{2}^{2}}+\frac{\partial^{2} u_{2}}{\partial x_{1} \partial x_{2}}\right)=0 \\
& \mu\left(\frac{\partial^{2} u_{1}}{\partial x_{1} \partial x_{2}}+\frac{\partial^{2} u_{2}}{\partial x_{1}^{2}}\right)+(2 \mu+\lambda) \frac{\partial^{2} u_{2}}{\partial x_{1}^{2}}+\lambda \frac{\partial^{2} u_{1}}{\partial x_{2} \partial x_{1}}-\frac{\partial P}{\partial x_{2}}=0 .
\end{aligned}
$$

The three equations (2.7), (2.12) and (2.13), for three unknown variables $\left(u_{1}\right.$, $u_{2}$ and $P$ ), are the governing poroelastic equations that model the interstitial deformation and the interstitial fluid flow.

\subsection{Boundary Conditions}

The periodicity of the geometry illustrated in Figure 2 allows us to reduce the problem to that on a single periodic tile containing one blood and one lymphatic capillary, see Figure 3. 


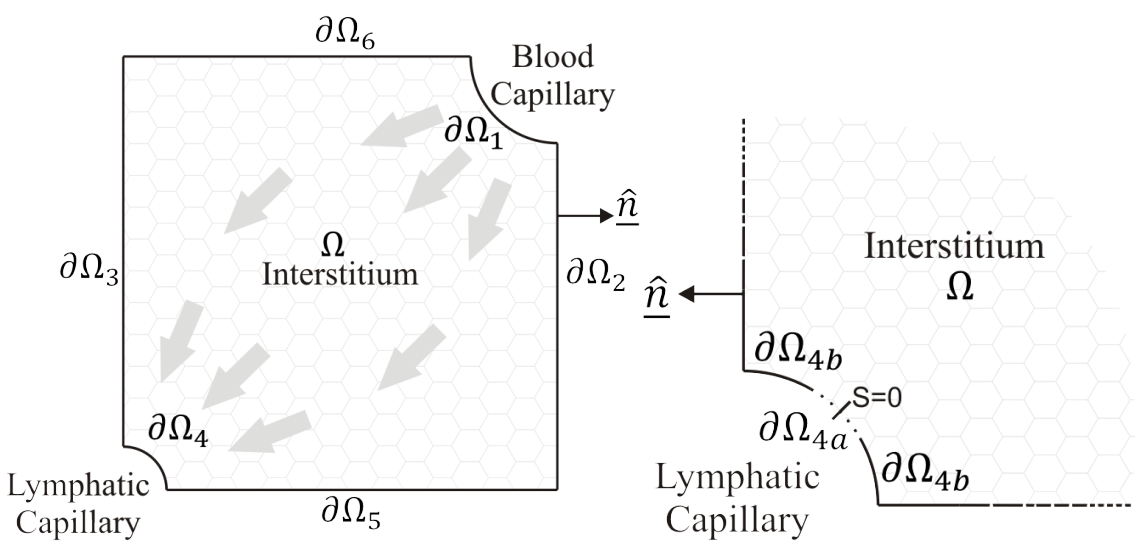

(a)

(b)

Figure 3: (a) The single periodic tile, containing one lymphatic and blood capillary separated by the interstitium. (b) A zoom of the lymphatic capillary identifying different parts of the capillary wall. Arrows in (a) indicate fluid motions.

\section{Evaluation of $\partial \Omega_{4 a}$ and $\partial \Omega_{4 b}$}

The lymphatic capillary wall $\left(\partial \Omega_{4}\right)$ is split into two parts, which are denoted by $\partial \Omega_{4 a}$ and $\partial \Omega_{4 b}$ (see Figure $3 \mathrm{~b}$ ). Here $\partial \Omega_{4 a}$ represents the interface between the lymphatic lumen and the interstitium not covered by lymphatic endothelial cells (through which the interstitial fluid flows), while $\partial \Omega_{4 b}$ represents the endothelial cell wall (through which there is no flow). We assume that the open section of the lymphatic capillary valve is located half way along the capillary boundary, as illustrated in Figure 3b. $C(t)$ is given by the length of the circumference of the lymphatic capillary

$$
C(t)=4 \int_{\partial \Omega_{4}} d s,
$$

and

$$
\begin{gathered}
\partial \Omega_{4 a} \text { is defined by } s \in[-\Delta, \Delta] \text { where } \Delta=\max \left\{0, \frac{C(t)-C_{\text {crit }}}{8}\right\}, \\
\partial \Omega_{4 b} \text { is defined by } s \in\left[-\frac{C(t)}{8},-\Delta\right] \text { and } s \in\left[\Delta, \frac{C(t)}{8}\right] .
\end{gathered}
$$

We note that $\partial \Omega_{4}$ is the union of $\partial \Omega_{4 a}$ and $\partial \Omega_{4 b}$, and when the lymphatic valve is closed $(\Delta=0) \partial \Omega_{4}=\partial \Omega_{4 b}$. With this in mind we calculate the length of the open section of the valve $\left(L_{v}(t)\right)$, in the relation,

$$
L_{v}(t)=\max \left\{0, \frac{C(t)-C_{c r i t}}{4}\right\},
$$

where $C_{\text {crit }}$ is the total length of the endothelial cells forming the valve (thus when $C<C_{\text {crit }}$ the valve is closed). We note that using (2.17) to determine the length 
of the open section of the valve uses the fact that the length of the endothelial cell wall $\left(\partial \Omega_{4 b}\right)$ does not change (while undergoing the 'sliding motion'). This assumption however does imply that the endothelial cell wall cannot deflect in the normal direction of the lymphatic capillary.

\subsubsection{Fluid Boundary Conditions}

\section{The Lymphatic capillary, boundary $\partial \Omega_{4}$}

The opening and closing of the lymphatic valve requires us to formulate two sets of fluid flow boundary conditions on the lymphatic valve. When the lymphatic valve is closed $\left(C(t)<C_{\text {crit }}\right)$ there is no normal fluid flow through the capillary wall relative to the tissue. Once the surrounding interstitium expands enough to open the lymphatic valve $\left(C(t)>C_{c r i t}\right)$, i.e., to slide the neighbouring lymphatic endothelial cells apart, fluid flow into the lumen occurs through the gap between the endothelial cells, i.e., through boundary $\partial \Omega_{4 a}$. We model this by imposing continuity of pressure on the open part of the lymphatic valve $\left(\partial \Omega_{4 a}\right)$ while keeping the no flux condition on the remaining part of the lymphatic wall $\left(\partial \Omega_{4 b}\right)$. Thus

$$
\begin{aligned}
P & =P^{l} \text { for } \quad \mathbf{x} \in \partial \Omega_{4 a}, \\
\nabla P \cdot \mathbf{n} & =0 \text { for } \quad \mathbf{x} \in \partial \Omega_{4 b},
\end{aligned}
$$

where $P^{l}$ is the fluid pressure of the lymphatic lumen and $\mathbf{n}$ is the unit normal vector $\left(\partial \Omega_{4}\right)$ pointing into the lumen.

\section{The blood capillary, boundary $\partial \Omega_{1}$}

The blood capillary wall is permeable to fluid flow, but provides a resistance to it. This behavior is modeled by imposing a linear permeability condition on the wall,

$$
K \nabla P \cdot \mathbf{n}=L_{p}\left(P-P^{b}\right) \quad \text { for } \quad \mathbf{x} \in \partial \Omega_{1},
$$

where $L_{p}$ is the vascular permeability and $\mathbf{n}$ is the unit normal vector to $\partial \Omega_{1}$ pointing into the capillary lumen.

Remaining edges of the periodic tile, boundaries $\partial \Omega_{2}, \partial \Omega_{3}, \partial \Omega_{5}$ and $\partial \Omega_{6}$

Due to the periodicity of the geometry the fluid only enters the interstitium through the blood capillary and only exits via the lymphatic capillary (once the valve has opened). Thus there is no fluid flux across the boundaries $\partial \Omega_{2}, \partial \Omega_{3}$, $\partial \Omega_{5}$ and $\partial \Omega_{6}$, namely

$$
\left(\mathbf{v}^{f}-\frac{\partial \mathbf{u}}{\partial t}\right) \cdot \mathbf{n}=0 \quad \text { for } \quad \mathbf{x} \in \partial \Omega_{2} \cup \partial \Omega_{3} \cup \partial \Omega_{5} \cup \partial \Omega_{6},
$$

where $\mathbf{n}$ is the unit outward normal vector to each edge. It follows that Darcy's equation (2.2) that,

$$
\nabla P \cdot \mathbf{n}=0 \quad \text { for } \quad \mathbf{x} \in \partial \Omega_{2} \cup \partial \Omega_{3} \cup \partial \Omega_{5} \cup \partial \Omega_{6} .
$$




\subsubsection{Solid Boundary Conditions}

Here we restrict our attention to uniform dilations (and contractions) of the periodic tile assuming that it remains square. This assumption disallows buckling of the lymphatic capillary and is reasonable for the small deformations considered in this paper. Since we are looking for a uniform dilation (or contraction) of the interstitium the edges of the periodic tile have to remain horizontal $\left(\partial \Omega_{2}\right.$ and $\left.\partial \Omega_{5}\right)$ and vertical $\left(\partial \Omega_{3}\right.$ and $\left.\partial \Omega_{6}\right)$.

\section{Edges adjacent to the blood capillary, boundaries $\partial \Omega_{2}$ and $\partial \Omega_{6}$}

Edges $\partial \Omega_{2}$ and $\partial \Omega_{6}$ of the periodic tile are only displaced in the tangential direction, keeping the tile square. Thus without loss of generality we impose zero normal displacement of boundaries $\partial \Omega_{2}$ and $\partial \Omega_{6}$. However, we allow boundaries $\partial \Omega_{2}$ and $\partial \Omega_{6}$ to deform freely in the tangential direction; which is equivalent to imposing zero tangential shear stress conditions. These two conditions can be formulated as

$$
\begin{aligned}
& u_{1}=0 \quad \text { and } \quad \sigma_{12}=0 \quad \text { for } \quad \mathbf{x} \in \partial \Omega_{2} \\
& u_{2}=0 \quad \text { and } \quad \sigma_{21}=0 \text { for } \quad \mathbf{x} \in \partial \Omega_{6} .
\end{aligned}
$$

Edges adjacent to the lymphatic capillary, boundaries $\partial \Omega_{3}$ and $\partial \Omega_{5}$

The symmetry of the problem requires that the tile remains square and, as a result, the edges $\partial \Omega_{3}$ and $\partial \Omega_{5}$ can deform freely in the tangential direction, but can only be displaced uniformly, by a prior unknown distance $u_{c}(t)$, which we shall calculate. These conditions can be formulated mathematically as

$$
\begin{aligned}
& u_{1}=u_{c}(t) \quad \text { and } \quad \sigma_{12}=0 \quad \text { on } \quad \mathbf{x} \in \partial \Omega_{3}, \\
& u_{2}=u_{c}(t) \quad \text { and } \quad \sigma_{21}=0 \quad \text { on } \quad \mathbf{x} \in \partial \Omega_{5},
\end{aligned}
$$

and the challenge now is how to compute $u_{c}(t)$.

In order to find an expression for $u_{c}(t)$ we first integrate the continuity equation (2.7) over $\Omega$, apply the divergence theorem, and make use of the boundary conditions (2.22) to obtain an expression for the boundary integral of the rate of change of the normal component of the matrix displacement on the boundary

$$
\frac{\partial}{\partial t} \int_{\partial \Omega} \mathbf{u} \cdot \mathbf{n} d S=\int_{\partial \Omega_{1} \cup \partial \Omega_{4}} K \nabla P \cdot \mathbf{n} d S, \quad \text { where } \partial \Omega=\bigcup_{i=1}^{6} \partial \Omega_{i} .
$$

Here we have made the assumption that tissue deformations are small so that $\int_{\partial \Omega} \boldsymbol{u}_{t} \cdot \boldsymbol{n} d S \approx(\partial / \partial t) \int_{\partial \Omega} \boldsymbol{u} \cdot \boldsymbol{n} d S$. The left hand side of equation (2.27) can also be written in the form

$$
\frac{\partial}{\partial t} \int_{\partial \Omega} \mathbf{u} \cdot \mathbf{n} d S=\frac{\partial}{\partial t}\left(\int_{\partial \Omega_{3} \cup \partial \Omega_{5}} \mathbf{u} \cdot \mathbf{n} d S+\int_{\partial \Omega_{1} \cup \partial \Omega_{4}} \mathbf{u} \cdot \mathbf{n} d S+\int_{\partial \Omega_{2} \cup \partial \Omega_{6}} \mathbf{u} \cdot \mathbf{n} d S\right)
$$


which, on using the boundary conditions (2.23)-(2.26) to simplify the first and eliminate the third integral on the right hand side, reduces to

$$
\frac{\partial}{\partial t} \int_{\partial \Omega} \mathbf{u} \cdot \mathbf{n} d S=\frac{\partial}{\partial t}\left(2 u_{c} L(t)\right)+\frac{\partial}{\partial t} \int_{\partial \Omega_{1} \cup \partial \Omega_{4}} \mathbf{u} \cdot \mathbf{n} d S
$$

where $L(t)$ is the width of the (square) periodic tile minus the radius of the lymphatic capillary. Substituting for the term of the left hand side of (2.28), from $(2.27)$, and integrating with respect to time gives the following expression for $u_{c}(t)$ :

$$
u_{c}(t)=\frac{1}{2 L(t)}\left(\int_{\partial \Omega_{1} \cup \partial \Omega_{4}}\left[\int K \nabla P \cdot \mathbf{n} d t\right] d S-\int_{\partial \Omega_{1} \cup \partial \Omega_{4}} \mathbf{u} \cdot \mathbf{n} d S\right) .
$$

\section{The blood and lymphatic capillary wall, boundaries $\partial \Omega_{1}$ and $\partial \Omega_{4}$}

The fluid pressure in the lumen of the blood and lymphatic capillaries exert a normal force on their walls. We assume that both capillary walls are not capable of exerting a significant elastic force so that the normal stress exerted by the solid matrix on each wall is balanced by their lumen fluid pressure. Furthermore, since we assume that lymphatic valves are allowed to slide freely, we require the tangential component of the solid stress on the capillary wall to be zero. These two conditions can be formulated as follows,

$$
\begin{aligned}
& \sigma_{i j} n_{j}=-P^{b} n_{i} \quad \text { for } \quad \mathbf{x} \in \partial \Omega_{1} \\
& \sigma_{i j} n_{j}=-P^{l} n_{i} \text { for } \quad \mathbf{x} \in \partial \Omega_{4}
\end{aligned}
$$

where $P^{b}$ and $P^{l}$ denote the lumen fluid pressure in the blood and lymphatic capillaries, respectively.

\subsubsection{Initial conditions}

We consider a problem in which the tissue is initially undeformed and at the uniform reference pressure zero, that is

$$
\left.P\right|_{t=0}=\left.u_{1}\right|_{t=0}=\left.u_{2}\right|_{t=0}=0 \quad \text { in } \Omega .
$$

We then track the subsequent evolution until the deformation on the lymphatic capillary wall is such that its circumference is $C(t)=C_{\text {crit }}$ and the valve starts to slide open. We then use the resulting configuration as the initial condition for a problem in which the valve is open. 


\subsection{Parameter Values}

There are 9 material parameter values in our governing equations and boundary conditions, namely $L_{p}, K, L_{i}, \mu, \lambda, P^{l}, P^{b}, C_{\text {crit }}, L_{\text {ovlap }}$; see Table 1 for estimates of their values.

The first and second Lamé constants, $\lambda$ and $\mu$, appear in (2.12) and (2.13). The elasticity of drained soft tissues $(2 \mu+\lambda)$ was measured by Swartz [54] to be in the range of $10^{4} \mathrm{~Pa}$ to $2 \times 10^{4} \mathrm{~Pa}$. The Poisson's ratio $(\nu)$ and the Young's Modulus $(E)$ are given in [26] for soft tissue as $\nu=0.35$ and $E=10^{4} \mathrm{~Pa}$. This gives $\lambda=8642 \mathrm{~Pa}$ and $\mu=3704 \mathrm{~Pa}$. We note that the Poisson ratio thus calculated is consistent with measurements made in $[4,47]$. With these parameter ranges $E$ is in the range $5 \times 10^{4} \mathrm{~Pa}$ to $1.3 \times 10^{5} \mathrm{~Pa}$, which is consistent with the studies by $[18,26]$.

For the hydraulic conductivity we choose a value of $5.1 \times 10^{-12} \mathrm{~m}^{3} \mathrm{skg}^{-1}$ in line with permeability value given in [2] of $7.6 \times 10^{-15} \mathrm{~m}^{2}$ and the viscosity measurement of interstitial fluid $1.5 \times 10^{-3} \mathrm{kgm}^{-1} \mathrm{~s}^{-1}$ conducted in [14] (hydraulic conductivity $=$ permeability/viscosity). This lies in the range of interstitial permeability measurements quoted in [34], namely $1.5 \times 10^{-19} \mathrm{~m}^{2}$ to $1.5 \times 10^{-14} \mathrm{~m}^{2}$ and lies close to measurements of the hydraulic conductivity, by two different methods, in a mousetail given by Swartz [54], namely $1.1 \times 10^{-12} \mathrm{~m}^{3} \mathrm{~s} \mathrm{~kg}^{-1}$ and $5.3 \times 10^{-13} \mathrm{~m}^{3} \mathrm{~s} \mathrm{~kg}^{-1}$. Extreme values of hydraulic conductivity are usually associated with disordered states and we will not deal with these here, but leave them to the following paper.

The vascular permeability of a blood capillary, $L_{p}$, was measured in various normal tissues by Jain et al [25], Levick [34], Pusenjak [41], Baxter and Jain [4] and Sarntinoranont et al [47] and estimated to be in the range $2.78 \times 10^{-11}$ $\mathrm{m}^{2} \mathrm{skg}^{-1}$ to $1.37 \times 10^{-9} \mathrm{~m}^{2} \mathrm{skg}^{-1}$. We take the average value $L_{p}=7.0 \times 10^{-10}$ $\mathrm{m}^{2} \mathrm{skg}^{-1}$.

The blood capillary pressure is normally in the range $2666 \mathrm{~Pa}$ to $4000 \mathrm{~Pa}$ $[7,25]$ and its radius $R_{b}$ is typically around $10 \mu \mathrm{m}$. Lymphatic lumen pressures have been measured by Lee [33] to be in the range up to $440 \mathrm{~Pa}$. Lymphatic capillaries normally have a radius in the range of $5 \mu \mathrm{m}$ to $15 \mu \mathrm{m}$ [42]. However, more recent studies show that in rare cases lymphatic capillaries can reach a large radius of $30 \mu \mathrm{m}[36,52]$. In this model we assume a lymphatic capillary radius of $10 \mu \mathrm{m}$. Therefore $C_{\text {crit }}=4 L_{\text {ovlap }}+2 \pi R_{l}=66.8 \mu \mathrm{m}$, where $R_{l}$ is the radius of the blood capillary and $L_{\text {ovlap }}=1 \mu \mathrm{m}$ is the length of the lymphatic endothelial cell overlap, ranging between $0.1 \mu \mathrm{m}$ to $2 \mu \mathrm{m}$ [30,31]. Measurements from Leak [31] show that the distance between lymphatic and blood capillaries can be up to $400 \mu \mathrm{m}$ apart. However, more recent studies [19, 48] found distances between lymphatic and blood capillaries from $50 \mu \mathrm{m}$ and $250 \mu \mathrm{m}$. Here we shall assume the the distance between the centres of the lymphatic and blood capillaries is initially $165 \mu \mathrm{m}$. It follows that the initial length of the edge of the periodic tile is $165 / \sqrt{2}=117 \mu \mathrm{m}$ and that $L_{i}$, the initial width of the tile minus the radius of the lymphatic capillary $R_{l}$, is $107 \mu \mathrm{m}$. 


\begin{tabular}{|c|c|c|c|c|}
\hline Parameter & Value & Units & Description & Source \\
\hline$L_{p}$ & $7.0 \times 10^{-10}$ & $\mathrm{~m}^{2} \mathrm{skg}^{-1}$ & The vascular permeability. & {$[4,25,47]$} \\
\hline$K$ & $5.07 \times 10^{-12}$ & $\mathrm{~m}^{3} \mathrm{skg}^{-1}$ & $\begin{array}{l}\text { Hydraulic conductivity of the in- } \\
\text { terstitium }\end{array}$ & {$[4,25,47]$} \\
\hline$L_{i}$ & 117 & $\mu \mathrm{m}$ & $\begin{array}{l}\text { The initial width of the periodic } \\
\text { tile, which is square, minus the } \\
\text { radius of the lymphatic capil- } \\
\text { lary. So } L(0)=L_{i} \text {. }\end{array}$ & {$[19,48]$} \\
\hline$R_{l}$ & 5 & $\mu \mathrm{m}$ & Blood capillary radius & {$[36,42,52]$} \\
\hline$R_{b}$ & 10 & $\mu \mathrm{m}$ & Lymphatic capillary radius & {$[19,48]$} \\
\hline$\nu$ & 0.35 & & The Poisson ratio & {$[26,47,54]$} \\
\hline$E$ & $10^{4}$ & $\mathrm{~Pa}$ & The Young's modulus & {$[26,54]$} \\
\hline$P^{l}$ & 100 & $\mathrm{~Pa}$ & Pressure in lymphatic lumen & [33] \\
\hline$P^{b}$ & 3333 & $\mathrm{~Pa}$ & Blood capillary pressure & {$[7]$} \\
\hline$C_{\text {crit }}$ & 66.8 & $\mu \mathrm{m}$ & $\begin{array}{l}\text { Critical lymphatic circumfer- } \\
\text { ence }\end{array}$ & {$[30,31]$} \\
\hline$L_{\text {ovlap }}$ & 1 & $\mu \mathrm{m}$ & $\begin{array}{l}\text { Lymphatic endothelial cell over- } \\
\text { lap }\end{array}$ & {$[30,31]$} \\
\hline
\end{tabular}

Table 1: Summary of dimensional parameters used in the model along with their references. Note the values of $E=\mu(3 \lambda+2 \mu) /(\lambda+\mu)$ and $\nu=\lambda /(2(\lambda+\mu))$ above correspond to $\lambda=8642$ and $\mu=3704$.

\subsection{Nondimensionalization}

We nondimensionalize the model to determine the key parameters in the problem.

We choose to scale length with $L$, the initial width of the periodic tile minus the radius of the lymphatic capillary and nondimensionalise the governing equations and boundary conditions by

$$
P=(2 \mu+\lambda) \bar{P}, \quad \sigma_{i j}=(2 \mu+\lambda) \bar{\sigma}_{i j}, \quad \mathbf{u}=L \overline{\mathbf{u}}, \quad t=\frac{L^{2}}{K(2 \mu+\lambda)} \bar{t} .
$$




\section{Dimensionless Model}

The dimensionless governing equations are (after dropping the bars),

$$
\begin{aligned}
\frac{\partial}{\partial t}\left(\frac{\partial u_{1}}{\partial x_{1}}+\frac{\partial u_{2}}{\partial x_{2}}\right) & =\frac{\partial^{2} P}{\partial x_{1}}+\frac{\partial^{2} P}{\partial x_{2}} \\
\frac{\partial^{2} u_{1}}{\partial x_{1}^{2}}+\frac{\partial^{2} u_{2}}{\partial x_{1} \partial x_{2}}-\widehat{\mu}\left(\frac{\partial^{2} u_{2}}{\partial x_{1} \partial x_{2}}-\frac{\partial^{2} u_{1}}{\partial x_{2}^{2}}\right) & =\frac{\partial P}{\partial x_{1}} \\
\frac{\partial^{2} u_{1}}{\partial x_{1} \partial x_{2}}+\frac{\partial^{2} u_{2}}{\partial x_{2}^{2}}+\widehat{\mu}\left(\frac{\partial^{2} u_{2}}{\partial x_{1}^{2}}-\frac{\partial^{2} u_{1}}{\partial x_{1} \partial x_{2}}\right) & =\frac{\partial P}{\partial x_{2}} .
\end{aligned}
$$

The dimensionless fluid flow boundary conditions on the lymphatic capillary are ${ }^{1}$,

$$
\begin{gathered}
P=\widehat{P}^{l} \quad \text { on } \quad \mathbf{x} \in \partial \Omega_{4 a}, \\
\nabla P \cdot \mathbf{n}=0 \quad \text { on } \quad \mathbf{x} \in \partial \Omega_{4 b} .
\end{gathered}
$$

On the blood capillary wall we have,

$$
\nabla P \cdot \mathbf{n}=R_{b}\left(P-\widehat{P}^{b}\right) \quad \text { on } \quad \mathbf{x} \in \partial \Omega_{1},
$$

and on the remaining edges of the periodic tile we have,

$$
\nabla P \cdot \mathbf{n}=0 \quad \text { on } \quad \mathbf{x} \in \partial \Omega_{2} \cup \partial \Omega_{3} \cup \partial \Omega_{5} \cup \partial \Omega_{6} .
$$

The dimensionless solid boundary conditions are,

$$
\begin{aligned}
& u_{2}=\sigma_{21}=0 \quad \text { on } \quad \mathbf{x} \in \partial \Omega_{2} \text {, } \\
& u_{1}=\sigma_{12}=0 \quad \text { on } \quad \mathbf{x} \in \partial \Omega_{6} \text {, } \\
& u_{1}=u_{c} \quad \text { and } \quad \sigma_{12}=0 \quad \text { on } \quad \mathbf{x} \in \partial \Omega_{3} \text {, } \\
& u_{2}=u_{c} \quad \text { and } \quad \sigma_{21}=0 \quad \text { on } \quad \mathbf{x} \in \partial \Omega_{5} \text {, }
\end{aligned}
$$

where $u_{c}$ is calculated by,

$$
u_{c}=\int_{\partial \Omega_{1} \cup \partial \Omega_{4}} \int \nabla P \cdot \mathbf{n} d t d S-\int_{\partial \Omega_{1} \cup \partial \Omega_{4}} \mathbf{u} \cdot \mathbf{n} d S .
$$

On the blood and lymphatic capillary wall we have,

$$
\begin{aligned}
& \sigma_{i j} n_{j}=-\widehat{P}^{b} n_{i} \quad \text { on } \quad \mathbf{x} \in \partial \Omega_{1}, \\
& \sigma_{i j} n_{j}=-\widehat{P}^{l} n_{i} \quad \text { on } \quad \mathbf{x} \in \partial \Omega_{4} \text {. }
\end{aligned}
$$

The dimensionless initial conditions are,

$$
\left.P\right|_{t=0}=\left.u_{1}\right|_{t=0}=\left.u_{2}\right|_{t=0}=0 \quad \text { in } \Omega .
$$

\footnotetext{
${ }^{1}$ See Table 2 for definitions of all the dimensionless parameter groupings.
} 
Table 2 shows the definitions and values of all the dimensionless parameters.

\begin{tabular}{|ccc|}
\hline Parameter & Definition & Estimated Value \\
\hline$\widehat{\mu}$ & $\frac{\mu}{2 \mu+\lambda}=\frac{1-2 \nu}{2(1-\nu)}$ & 0.23 \\
$R_{b}$ & $\frac{L L_{p}}{K}$ & 0.016 \\
$\widehat{P}^{b}$ & $\frac{P^{b}}{2 \mu+\lambda}=\frac{P^{b}(1+\nu)(1-2 \nu)}{E(1-\nu)}$ & 0.21 \\
$\widehat{P}^{l}$ & $\frac{P^{l}}{2 \mu+\lambda}=\frac{P^{l}(1+\nu)(1-2 \nu)}{E(1-\nu)}$ & 0.02 \\
\hline
\end{tabular}

Table 2: The values of the dimensionless groupings.

Dependence of the steady state solution on $\widehat{P}^{b}$ and $\widehat{P}^{l}$. We note that the steady state solution satisfies (2.38)-(2.47) and that these equations are invariant under the transformation

$$
\begin{aligned}
& P \rightarrow P+c, \quad \widehat{P}^{b} \rightarrow \widehat{P}^{b}+c, \quad \widehat{P}^{l} \rightarrow \widehat{P}^{l}+c, \quad \mathbf{u} \rightarrow \mathbf{u}, \\
& \sigma_{11} \rightarrow \sigma_{11}-c, \quad \sigma_{12} \rightarrow \sigma_{12}, \quad \sigma_{21} \rightarrow \sigma_{21}, \quad \sigma_{22} \rightarrow \sigma_{22}-c,
\end{aligned}
$$

where $c$ is an arbitrary constant. Such a transformation leaves the Darcy flow in the interstitium $-\nabla P$ and the tissue deformation $\mathbf{u}$ unchanged and thus leads to a physically identical solution. It is thus clear that the steady state solution does not depend on lymphatic lumen pressure $\widehat{P}^{b}$ and blood capillary pressure $\widehat{P}^{l}$ (neither of which are invariant under the transformation), but depends solely on the pressure difference $\widehat{P}^{b}-\widehat{P}^{l}$ (which is invariant under the transformation).

\section{Solution and Results}

We numerically solve the model (2.7)-(2.8), (2.12)-(2.16), (2.18)-(2.26) and (2.29)(2.32), with the parameter values given in Table 1, using the Comsol Multiphysics Finite element package and display solutions in Figures 4-7.

We produce plots of the interstitial deformation to demonstrate three key stages of the drainage process. In Figure 4, we plot the interstitial deformation at times during the drainage process (the arrows in each plot indicate the direction of the fluid flow). In Figure 4a, the lymphatic valve is closed and since the inflowing interstitial fluid cannot escape the interstitium it causes it to deform, resulting in the boundaries of the periodic tile moving outwards. At a later stage in the process (Figure 4b), the interstitial expansion about the lymphatic capillary is sufficiently large to cause the lymphatic endothelial cells to slide apart creating a gap for the interstitial fluid to drain into the lymphatic lumen. For sufficiently small gaps, the resistance to drainage is sufficiently large so that the net flow into the interstitium remains positive and the solid matrix keeps expanding. Once the interstitial deformation is large enough (Figure 4c), the gap length of the open section of the valve becomes sufficient to lower the resistance to drainage to a 
level where the drainage rate through the lymphatic valve exceeds the fluid inflow (from the blood capillary), and the solid matrix begins to contract. The gap length has peaked and the interstitium contracts until the lymphatic endothelial cells slide together again, closing the lymphatic valve.

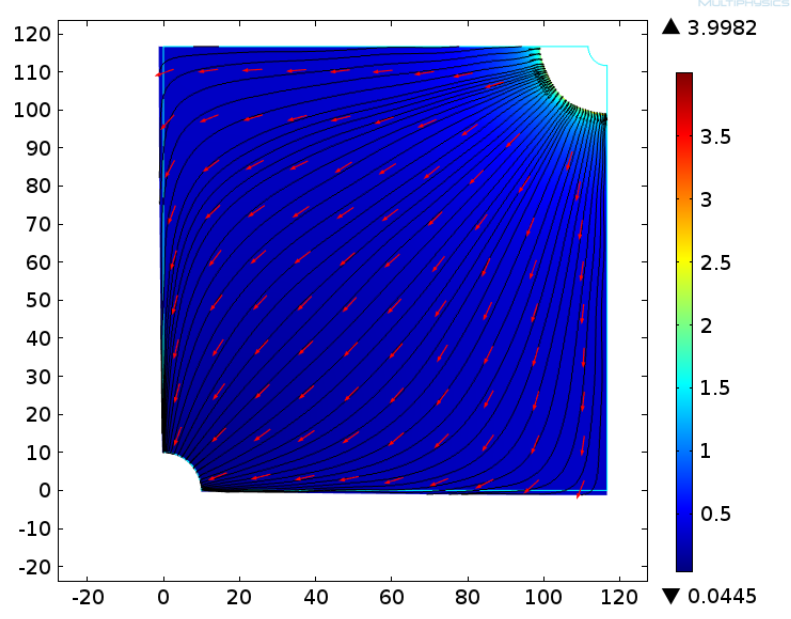

(a)

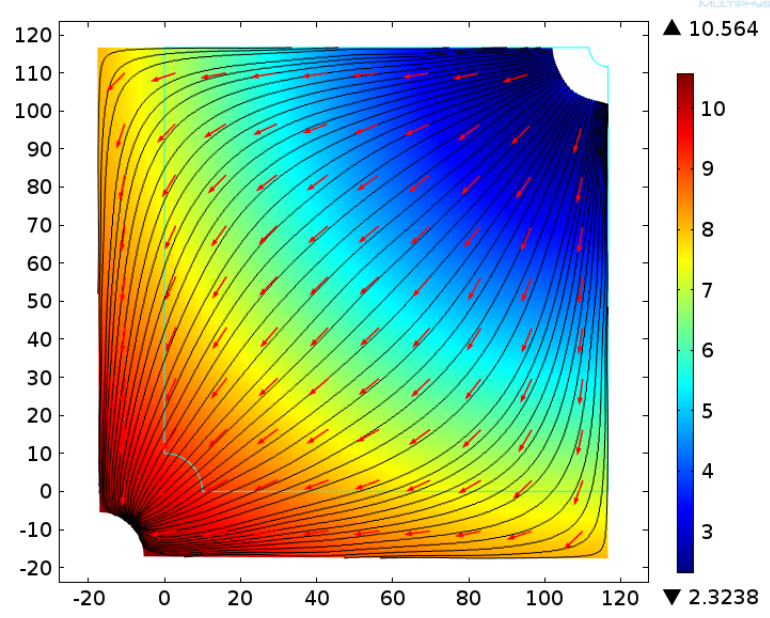

(c)

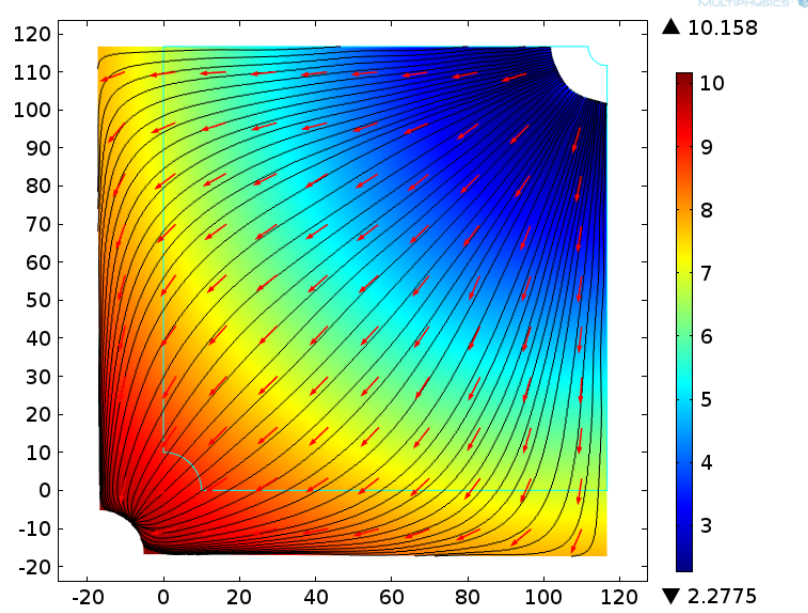

(b)

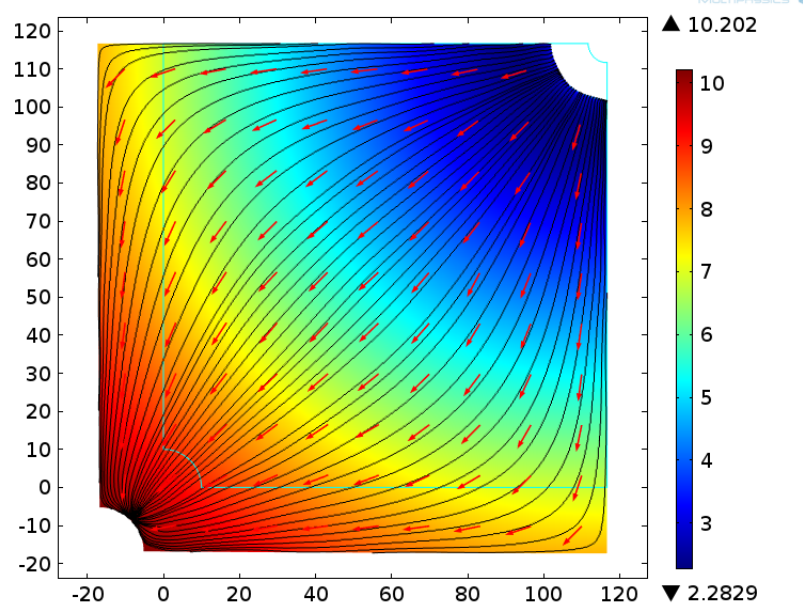

(d)

Figure 4: Deformations of the (solid) interstitial matrix $\boldsymbol{u}(x, y)$ (axes labelled in $\mu \mathrm{m}$ ). The colour map indicates the magnitude of the deformations in $\mu \mathrm{m}$, the black lines are the streamlines and arrows show the direction of fluid flow. The undeformed region in each plot is marked by a pale blue line. (a) The initial deformation of the solid matrix with the lymphatic valve closed. (b) The deformation of the solid matrix once the lymphatic endothelial cells first side apart $\left(C(t)>C_{c r i t}\right)$. (c) The maximum expansion of the solid matrix before it starts to contract. (d) The deformation of the interstitial matrix once the system has relaxed to steady state. 
Magnifications, about the lymphatic capillary, of the results shown in Figures 4a-4d are produced in Figures 5a-5d, respectively.

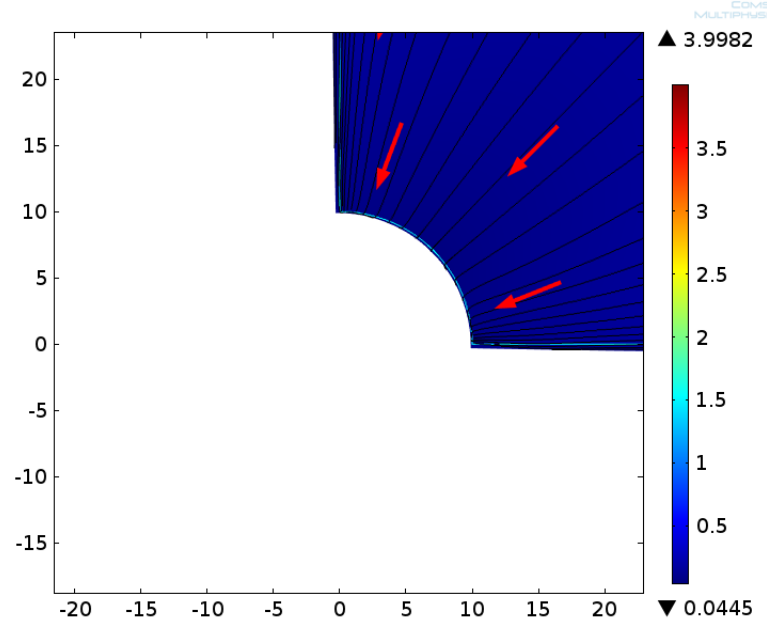

(a)

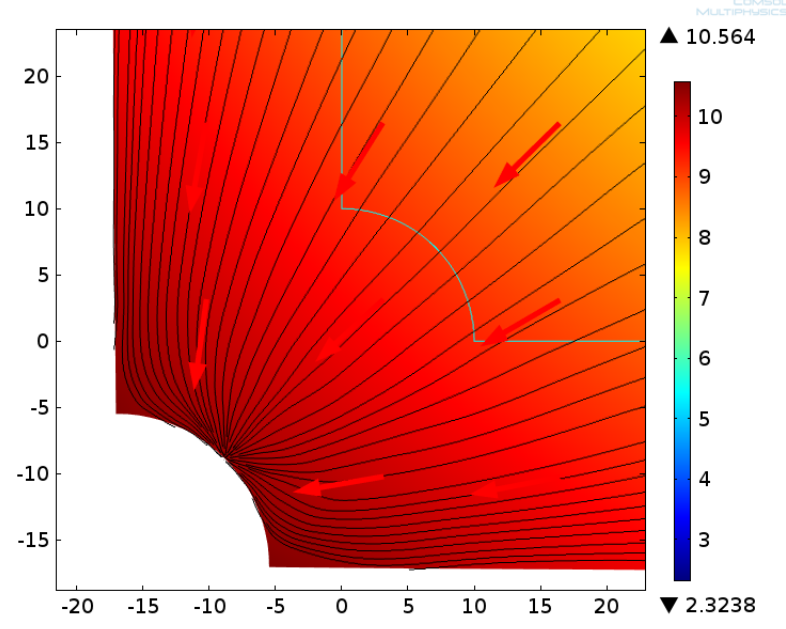

(c)

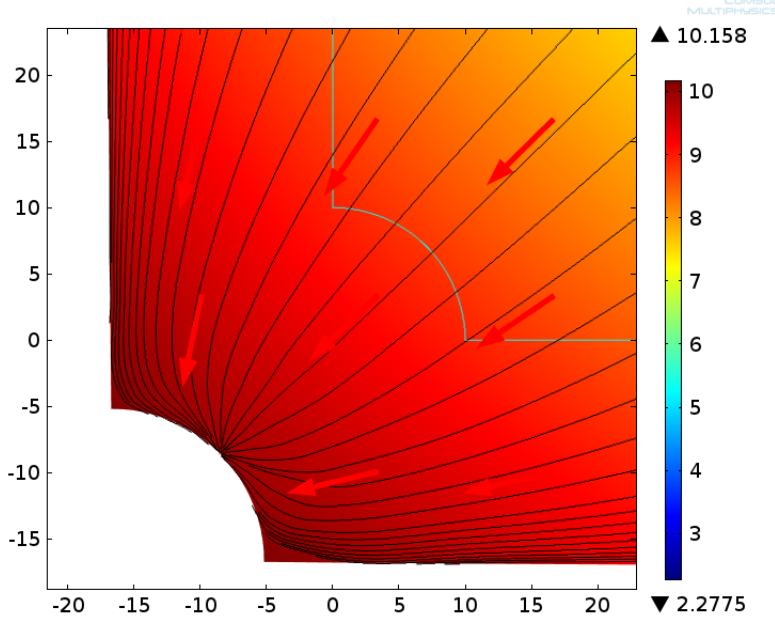

(b)

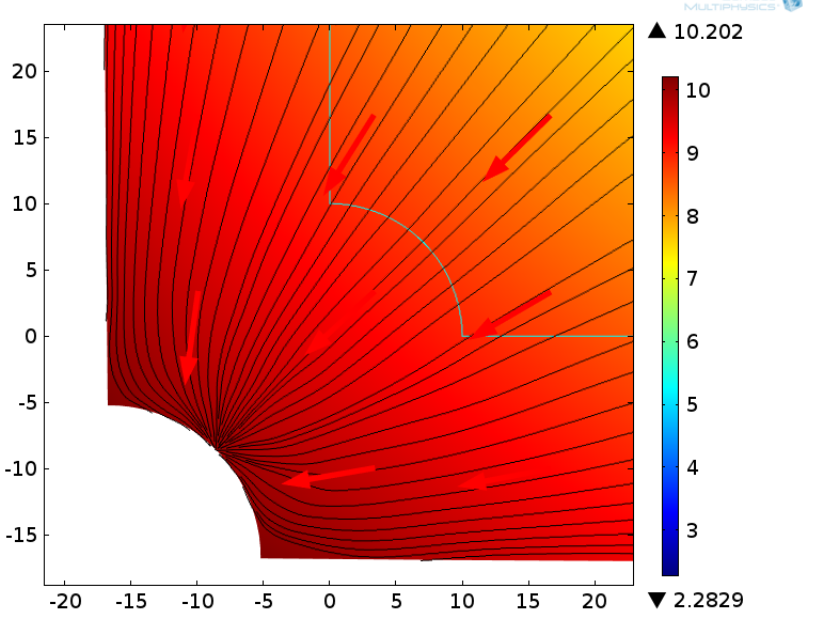

(d)

Figure 5: A magnification of figure 4 about the lymphatic capillary (axes labelled in $\mu \mathrm{m})$.

These plots show how the flow in the vicinity of the lymphatic valve changes as the valve opens. Since it is still hard to see the sliding action of the lymphatic valve, we have sketched the three key stages of the lymphatic valve opening in the sliding door fashion in Figure 6. 


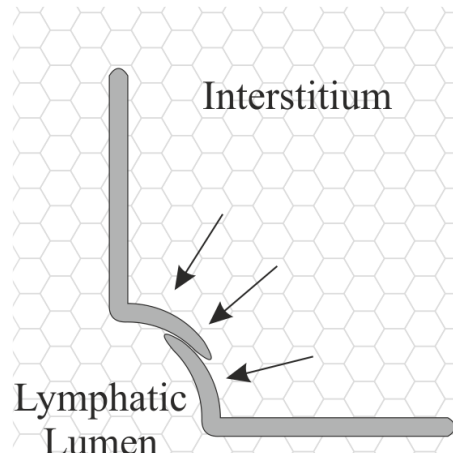

Lumen

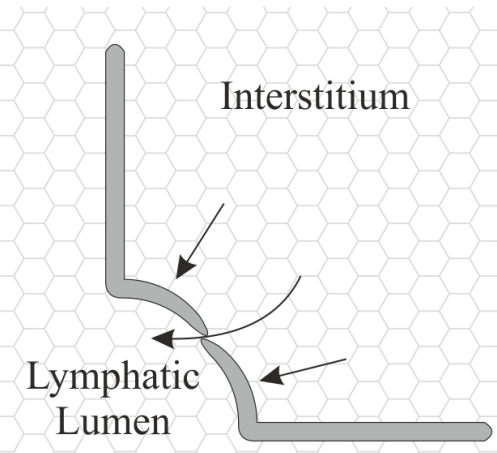

Lumen

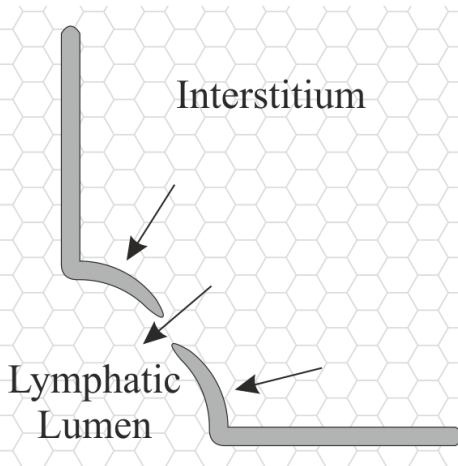

Figure 6: A drawing of the three stages described earlier that the lymphatic valve undergoes in draining interstitial fluid.

The gap length of the open section of the lymphatic valve is initially zero in Figure 7a (when the valve is closed). It remains closed until enough fluid enters the interstitium to cause an expansion that slides open the valve (this happens at $t=128 \mathrm{~s})$. The length of this gap increases until the outflow into the lymphatics exceeds the inflow from the blood capillary. Then the gap length decreases until the lymphatic valve closes. In this first cycle the gap length reached a maximum length of $0.045 \mu \mathrm{m}$. After two valve cycles the interstitium relaxes into a steady state with constant gap length of approximately $0.01 \mu \mathrm{m}$.

The Darcy flux into the lymphatic lumen was also calculated (Figure 7b). This is initially zero when the valve is closed and first becomes non zero when the valve opens $(t=128 \mathrm{~s})$. Integrating the velocity around the capillary gives us the Darcy flux into the lumen. It reaches a maximum of $9.74 \times 10^{-11} \mathrm{~m}^{2} / \mathrm{s}$ when the gap length is at its maximum in the first valve cycle. After two valve cycles the interstitium relaxes to a steady state, with constant Darcy flux of $0.35 \times 10^{-11} \mathrm{~m}^{2} / \mathrm{s}$. This corresponds to a net Darcy flux per unit length of lymphatic capillary of $1.41 \times 10^{-11} \mathrm{~m}^{2} / \mathrm{s}$, since we hypothesize four valves around the edge of the capillary. 


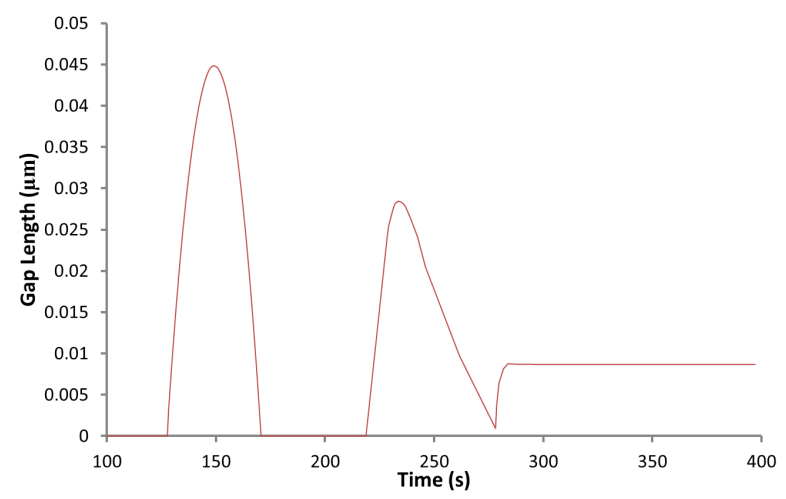

(a)

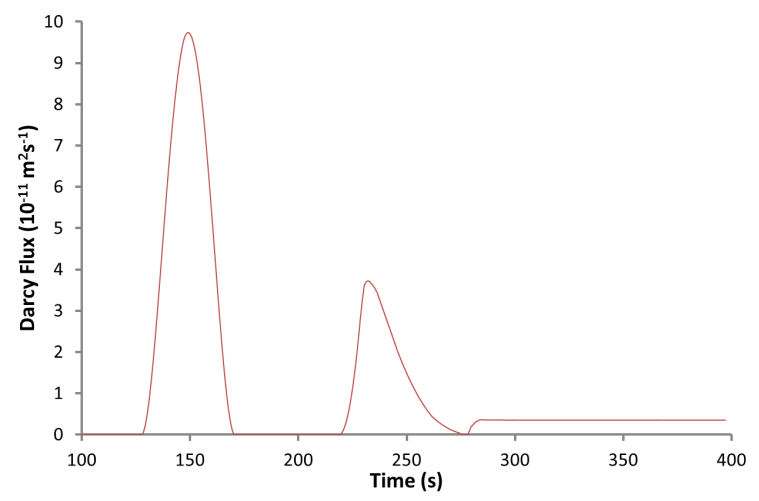

(b)

Figure 7: (a) This plot displays how the length of the open section of the lymphatic valve changes over time. (b) This plot displays the Darcy flux into the lymphatic lumen against time.

\subsection{Parameter Influence}

Here we investigate the role of parameter variations on lymphatic drainage by independently varying: (i) Young's modulus, (ii) pressure difference, (iii) vascular permeability, and (iv) initial length of lymphatic endothelial cell overlap in a series of numerical experiments. In Table 3 we provide a key that can be used to interpret the results of the numerical simulations presented in Figures 8-11. In all of these figures, as the parameter of interest is varied, we plot the steady state Darcy flux through the interstitium (from blood capillary to lymphatic capillary) and the total steady state area of the tissue as a proportion of the undeformed area of the tissue. These quantities are both physiologically important, particularly the latter which gives a measure of tissue swelling, and thus also of oedema.

\begin{tabular}{|l|l|l|l|l|}
\hline & $\begin{array}{l}\text { Young's } \\
\text { modulus } \\
E\end{array}$ & $\begin{array}{l}\text { Pressure } \\
\text { Difference } \\
P^{b}-P^{l}\end{array}$ & $\begin{array}{l}\text { Vascular Perme- } \\
\text { ability } L_{p}\end{array}$ & $\begin{array}{l}\text { Endothelial } \\
\text { cell overlap } \\
L_{\text {ovlap }}\end{array}$ \\
\hline Figure 8 & Varying & $3233 \mathrm{~Pa}$ & $7 \times 10^{-10} \mathrm{~m}^{2} \mathrm{skg}^{-1}$ & $1 \mu \mathrm{m}$ \\
Figure 9 & $10^{4} \mathrm{~Pa}$ & Varying & $7 \times 10^{-10} \mathrm{~m}^{2} \mathrm{skg}^{-1}$ & $1 \mu \mathrm{m}$ \\
Figure 10 & $10^{4} \mathrm{~Pa}$ & $3233 \mathrm{~Pa}$ & Varying & $1 \mu \mathrm{m}$ \\
Figure 11 & $10^{4} \mathrm{~Pa}$ & $3233 \mathrm{~Pa}$ & $7 \times 10^{-10} \mathrm{~m}^{2} \mathrm{skg}^{-1}$ & Varying \\
\hline
\end{tabular}

Table 3: A key to the parameter variations in Figures 8-11 (all other parameters as in Table 1).

A brief summary of the results plotted in Figures 8-11 is made below in Table 4 which shows how the steady state Darcy flux and the gap length are affected as 
the model parameters are varied.

\begin{tabular}{|cl|l|l|l|l|}
\hline & & $\begin{array}{l}\text { Steady } \\
\text { State } \\
\text { Flux }\end{array}$ & $\begin{array}{l}\text { Tissue } \\
\text { Expan- } \\
\text { sion }\end{array}$ & $\begin{array}{l}\text { Steady } \\
\text { State } \\
\text { Gap Size }\end{array}$ & $\begin{array}{l}\text { Time to } \\
\text { Steady } \\
\text { State }\end{array}$ \\
\hline Young's Modulus $E$ & $30 \% \uparrow$ & $18 \% \downarrow$ & $0.2 \% \downarrow$ & $13 \% \downarrow$ & $62 \% \downarrow$ \\
& $50 \% \downarrow$ & $50 \% \uparrow$ & $0.8 \% \uparrow$ & $81 \% \uparrow$ & $275 \% \uparrow$ \\
Pressure Difference $P^{b}-P^{l}$ & $24 \% \uparrow$ & $50 \% \uparrow$ & $5 \% \uparrow$ & $50 \% \uparrow$ & $52 \% \uparrow$ \\
& $100 \% \downarrow$ & $100 \% \downarrow$ & $12 \% \downarrow$ & $100 \% \downarrow$ & $100 \% \downarrow$ \\
Vascular Permeability $L_{p}$ & $43 \% \uparrow$ & $46 \% \uparrow$ & $6 \% \uparrow$ & $57 \% \uparrow$ & $75 \% \uparrow$ \\
& $100 \% \downarrow$ & $100 \% \downarrow$ & $11 \% \downarrow$ & $100 \% \downarrow$ & $100 \% \downarrow$ \\
Overlap Length $L_{\text {ovlap }}$ & $100 \% \uparrow$ & $55 \% \downarrow$ & $9 \% \uparrow$ & $65 \% \downarrow$ & $210 \% \uparrow$ \\
& $75 \% \downarrow$ & $65 \% \uparrow$ & $4 \% \downarrow$ & $110 \% \uparrow$ & $97 \% \downarrow$ \\
\hline
\end{tabular}

Table 4: Effects of varying the parameters independently on the steady state Darcy flux and the tissue expansion

Young's Modulus. The Young's modulus is an important measure of the physiological state of a tissue. It is known for example that tissue elasticity decreases with old age [12, 28, 49], pregnancy [27] and obesity [10,56]. The effects of varying the Young's modulus on the fluid flux into the lymphatic lumen are shown in Figure 8. As might be expected increases in stiffness (corresponding to an increase in Young's modulus) lead to significant decreases in the fluid flux through the interstitium and a decrease in time to reach steady state. Perhaps somewhat surprisingly increases in Young's modulus result only in a very slight decrease in the ratio of the steady-state tissue area to the undeformed area. However this may be explained by noting that a sliding valve opens in response to tissue deformation (rather than pressure difference) and so acts to control tissue dilatation. 


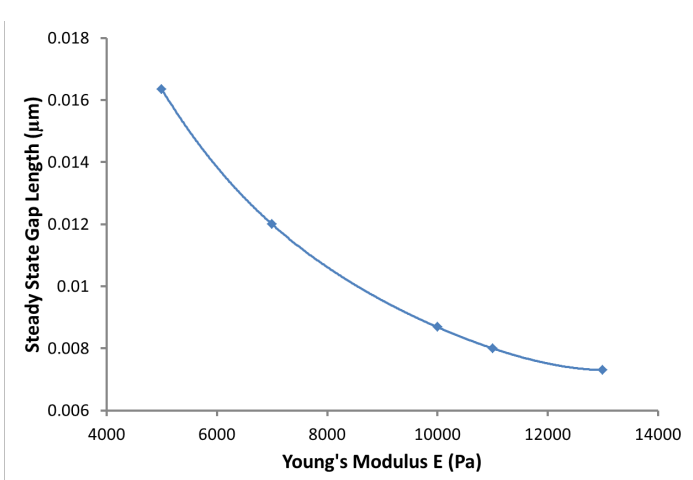

(a)

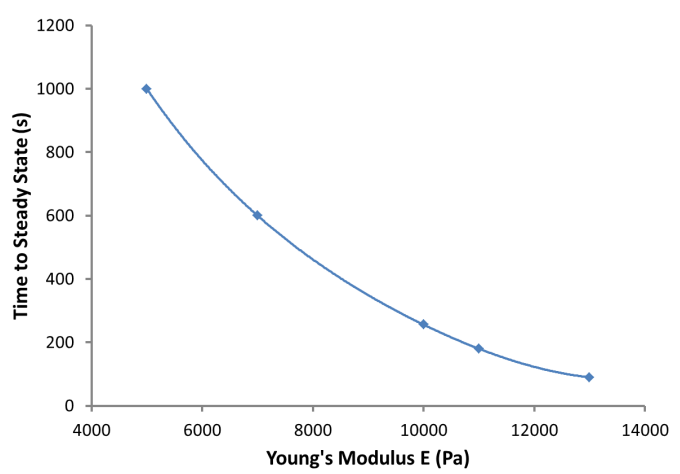

(c)

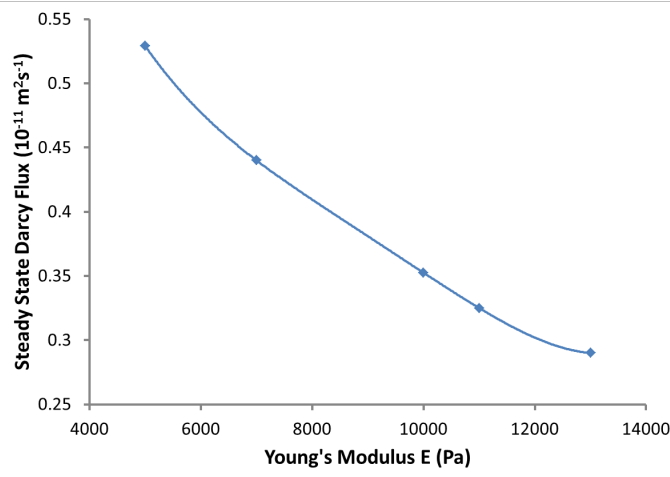

(b)

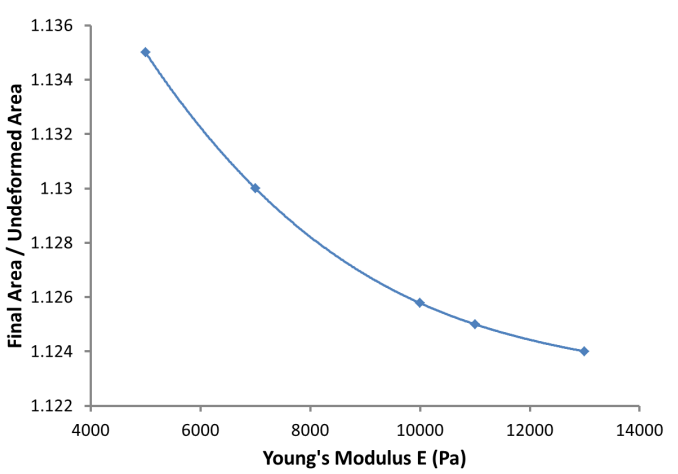

(d)

Figure 8: The effects of changes to the Young's modulus on (a) the steady state gap length of the open section of the lymphatic valve, (b) the steady state Darcy flux into the lymphatic lumen, (c) the time it taken to relax to the steady state, and (d) the degree of swelling at equilibrium (equilibrium area/undeformed area).

Pressure Difference. Increases in blood pressure in hypertensive patients do not tend to result in increases in the pressure difference, $P^{b}-P^{l}$, between the blood capillaries and the lymphatic capillaries [9] (smooth muscles in the upstream arterial network regulate the flow to protect the capillary bed) increased venous pressure (due to, for example, obstruction) can result in increase in $P^{b}-P^{l}[9]$.

The effects of varying the pressure difference, $P^{b}-P^{l}$, on the fluid flux into the lymphatic lumen are shown in Figure 9. As might be expected physiological increases in the pressure difference result in a significant increase in the Darcy flow through the interstitium, but also in small but noticable increase in the steady state dilatation of the tissue (as measured by the ratio of the steady-state tissue area to undeformed tissue area). 


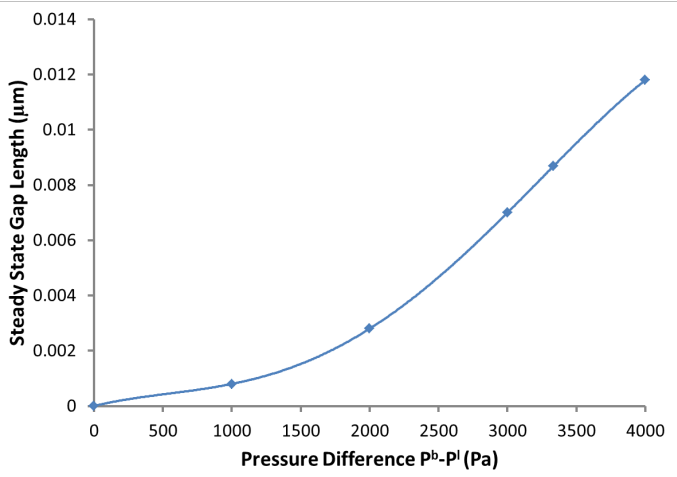

(a)

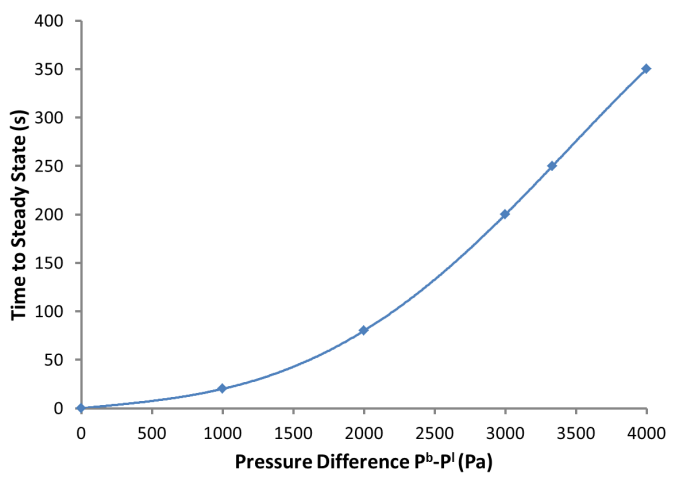

(c)

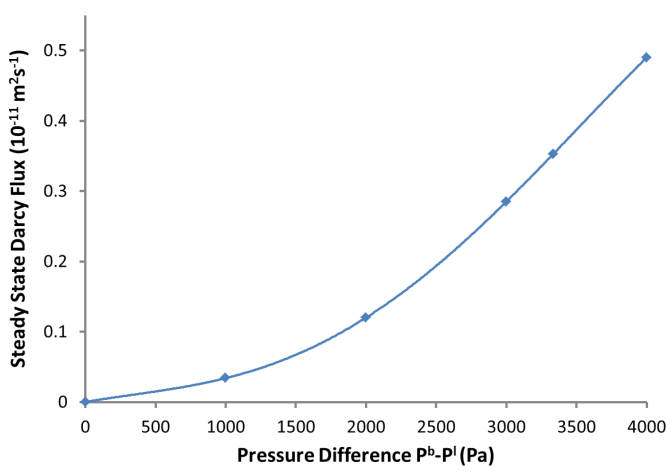

(b)

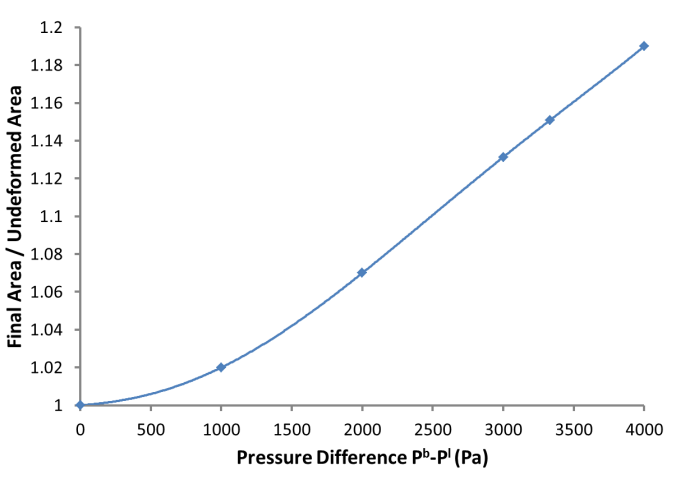

(d)

Figure 9: The effects of changes to the pressure difference between the vascular and lymphatic capillaries on (a) steady state gap length of the open section of the lymphatic valve, (b) steady state Darcy flux into the lymphatic lumen, (c) time taken to relax to steady state, and (d) the degree of swelling at equilibrium (equilibrium area/undeformed area).

Vascular Permeability. The effects of varying the vascular permeability on the fluid flux into the lymphatic lumen are shown in Figure 10. Of particular note is that physiological increases in vascular permeability result in significant increases in the flow through the interstitium (Figure 10b), but that the same increase in permeability result in only slight increases in tissue deformation (Figure 10d). Once again, as in the case of the Young's modulus, the small change in tissue deformation with increase in permeability may be attributed to the fact that it is the interstitial expansion that is responsible for opening the sliding valve. Clinical studies show that in obesity [16], pregnancy [1, 35] and in the elderly [17, 29] vascular permeability increases. Furthermore that cases of oedema are common in patients who have a high vascular permeability $[3,51]$. This might appear to be at odds with the results of our model. However we note that increases in vascular permeability may also be associated with the entry of white blood cells and other large objects into the interstitium that can act to block fluid drainage paths and cause swelling. In addition other parts of the lymphatic system may be unable 
to cope with the large fluxes flowing through the lymphatic capillaries. Another possibility, that should perhaps be considered in future, is that real valves may actually be activated by a combination of tissue dilatation and pressure differences across the lining of the lymphatic capillaries.

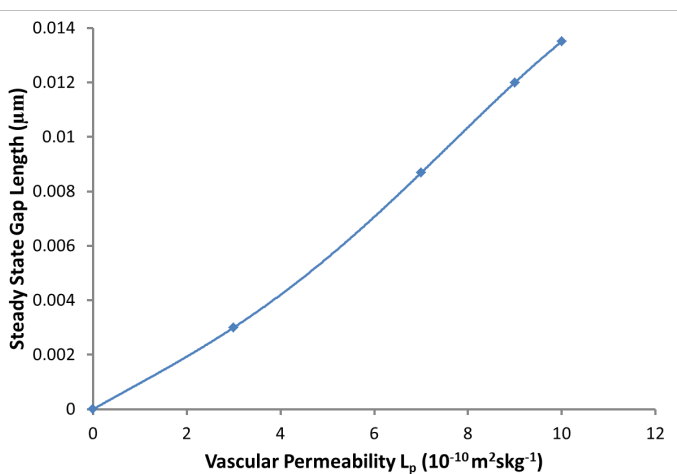

(a)

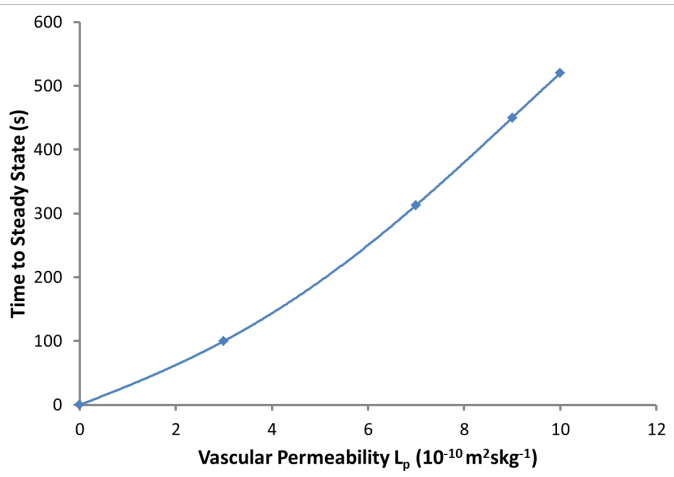

(c)

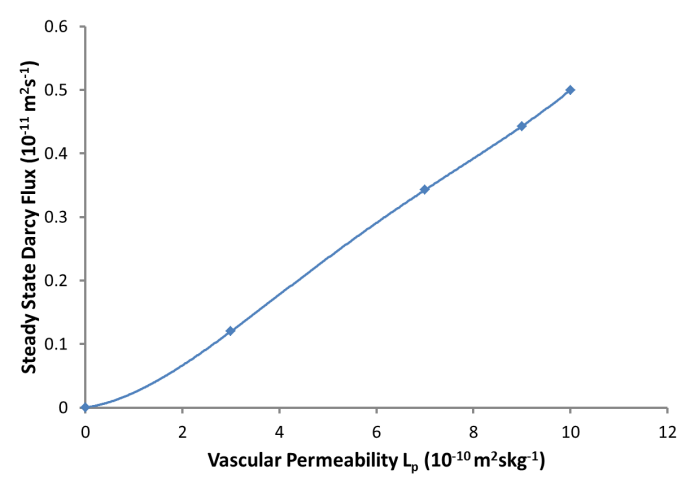

(b)

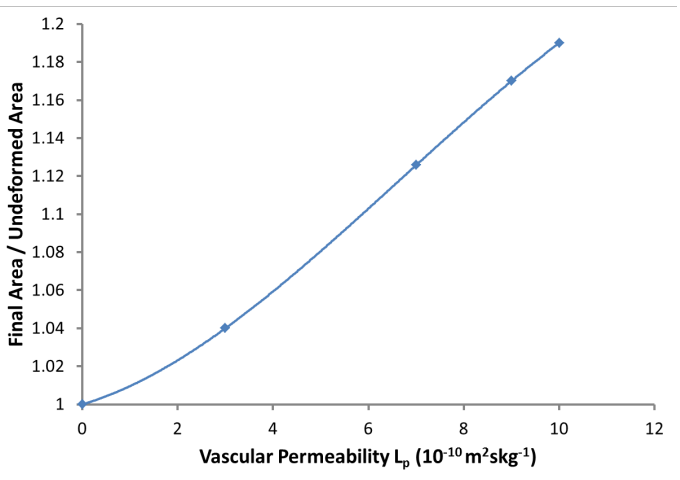

(d)

Figure 10: The effects of changes to the vascular permeability to (a) the steady state gap length of the open section of the lymphatic valve, (b) the steady state Darcy flux into the lymphatic lumen, (c) the time taken to relax to steady state and (d) the degree of swelling at equilibrium (equilibrium area/undeformed area).

Length of the Lymphatic Endothelial Cell Overlap. The effects of varying the length of the lymphatic endothelial cell overlap on the fluid flux into the lymphatic lumen are shown in Figure 11. The lymphatic endothelial cell overlap $\left(C_{\text {crit }}-C(0)\right)$ values fluctuate for different lymphatic capillaries throughout the body, most ranging between $0.1 \mu \mathrm{m}$ to $2 \mu \mathrm{m}$ [30, 31]. As shown in Figure 11(d), increasing this length increases the tissue dilatation; this is because it takes a greater local interstitial deformation to slide open the valve. As also might be expected the fluid flux through the interstitium (figure 11b) also decreases with increases in overlap length. 


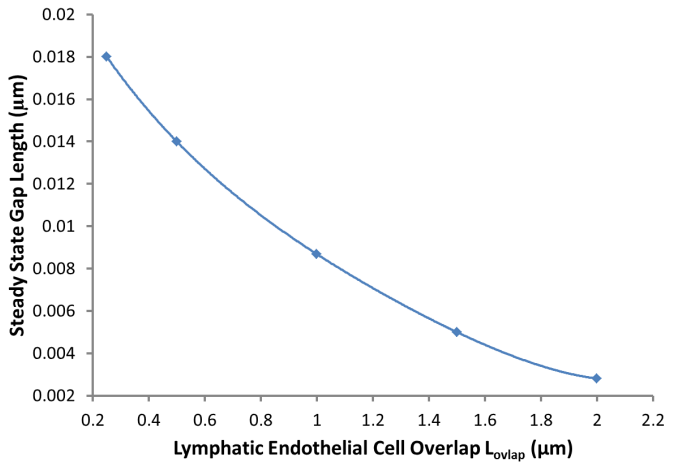

(a)

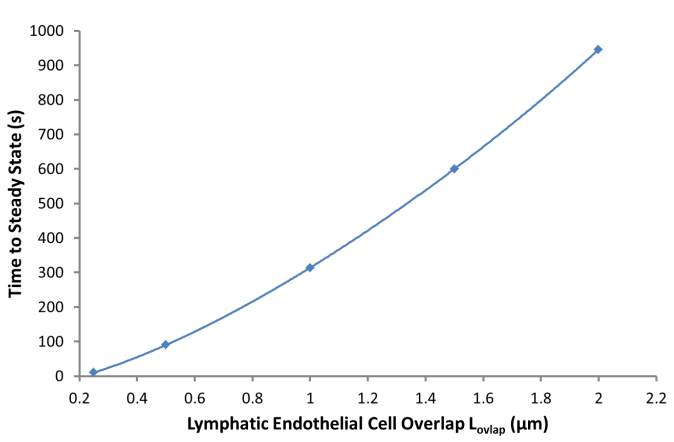

(c)

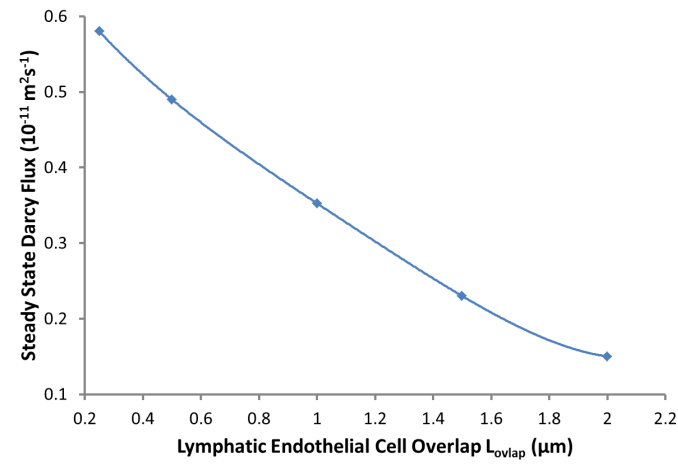

(b)

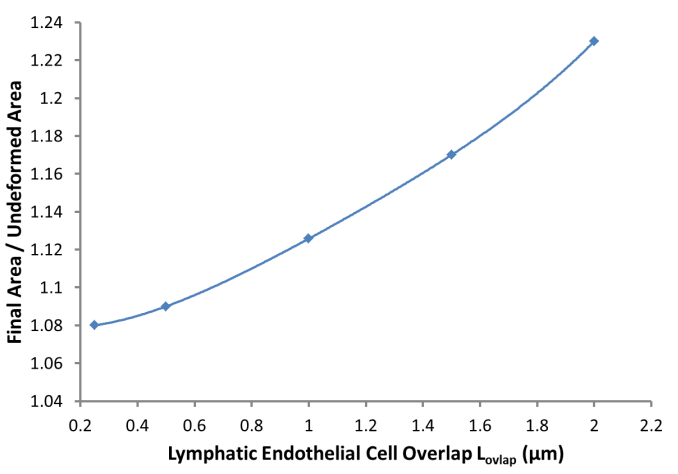

(d)

Figure 11: Exploring the effects of changes to the overlap length on the drainage behaviour. (a) The steady state gap length of the open section of the lymphatic valve. (b) The steady state Darcy flux into the lymphatic lumen. (c) The time it takes to relax to steady state. (d)the degree of swelling at equilibrium (equilibrium area/undeformed area).

We have been unable to find physiological evidence, in the literature, that lymphatic endothelial cell overlap length varies in different disease states. However it does vary in different organs, but there seems to be no physiological evidence that it changes in old age, pregnancy and obesity.

\subsection{Fluctuation to the Lymphatic Lumen Pressure.}

The lumen pressure in the primary lymphatic system fluctuates as a consequence of pressure variations in the secondary lymphatic system $[15,55,57]$. In order to investigate these fluctuations in lymphatic lumen pressure we apply a periodic lymphatic pressure $P^{l}$, after the system has been allowed to equilibriate, and investigate its effects on primary lymphatic drainage. We did this by taking the lymphatic lumen pressure equals $100 \mathrm{~Pa}$ for $t<300 \mathrm{~s}$ and $100(1+\sin (2 \pi t / n)) \mathrm{Pa}$ for $t>300 \mathrm{~s}$ (where the period $n=2 \mathrm{~s}$ ) and, as in the previous dynamic simulation, we start the tissue from its undeformed state at $t=0$. This profile was chosen lumen pressure fluctuations commence only after the system has relaxed to a 
steady state. The results plotted in Figure 12 show that, as might be expected, these physiological pressure fluctuations in lymphatic pressure cause only very minor perturbations to the steady state and this strongly suggests that it is the properties of the steady state that are of importance.

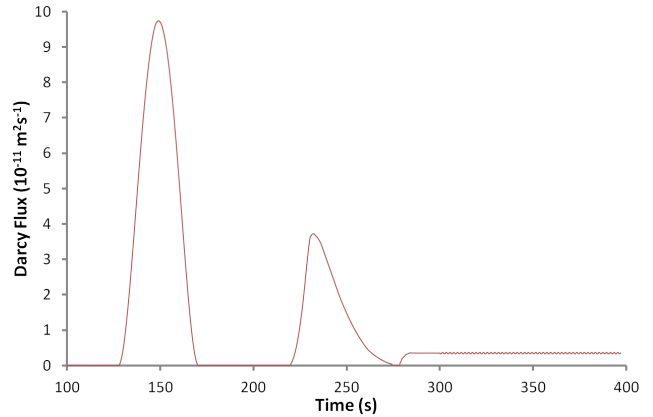

(a)

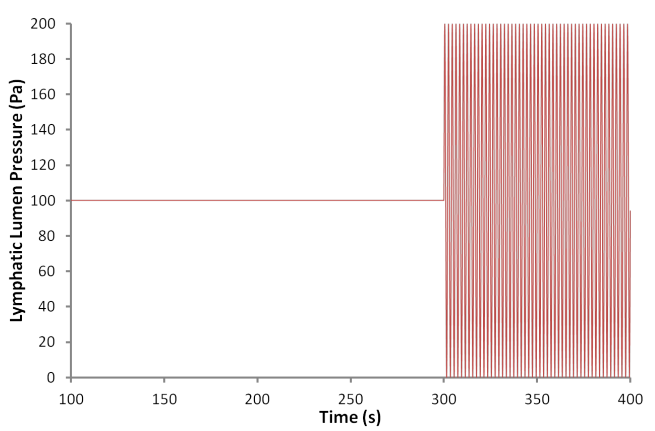

(c)

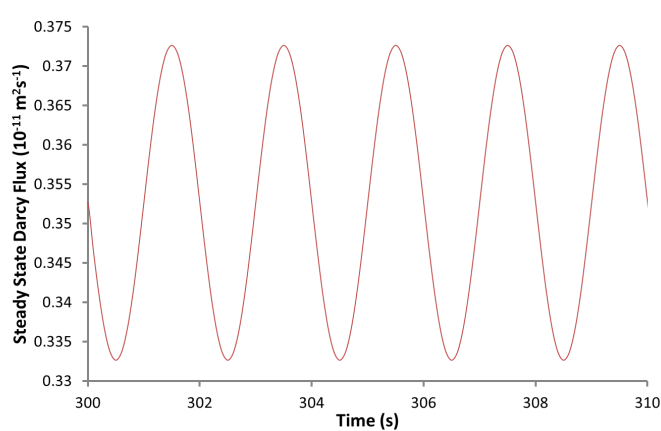

(b)

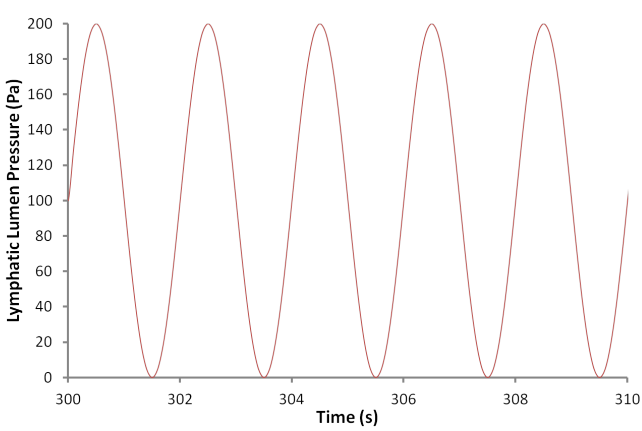

(d)

Figure 12: The effect of oscillating the lymphatic lumen pressure on the drainage behaviour. (a) Darcy Flux against time (b) Oscillations of the Darcy flux about the equilibrium (at long times). (c) Lymphatic lumen pressure as function of time. (d) Oscillations of lymphatic lumen pressure at long times.

\section{Discussion}

Around $20 \%$ of the body's mass is thought to be interstitial fluid [34]. An understanding the mechanics of the lymphatic system and its role in interstitial drainage is therefore of significant physiological importance. In contrast to earlier studies [11, 18, 38, 42, 43, 44, 45], in which lymphatic valves are presumed to open in response to fluid pressure differences between the interstitium and lymphatic lumen, this work outlines a hypothesis for lymphatic drainage of interstitial fluid which is based upon the Rossi conjecture [46]. This presumes that endothelial gap junctions on the lymphatic capillary walls are pulled open in response to swelling of the surrounding tissue (which would occur, for example, when fluid enters the interstitium from the blood capillaries). In order to investigate this conjecture we formulated a model for this process in which elastic deformations of the tissue, 
and the flow of fluid through the interstitium, are treated using Biot's equations of poroelasticity. The fluid flux into the interstitium from the blood capillaries is assumed to be linearly proportional to the pressure difference between the blood capillary lumen pressure and that in the surrounding tissue (the constant of proportionality being the vascular permeability). We modelled the flow from interstitium into the lymphatic lumen based on Rossi's conjecture by assuming (I) no flow into the lumen when the circumference of the lymphatic capillary is below a certain critical size (the overlapping endothelial cells form a barrier) and (II) a flow that is controlled by the gap lengths left between adjacent endothelial cells when the surrounding tissue has swelled sufficiently to stretch the capillary's circumference above the critical size.

Solutions to the model were obtained in an idealized 2D periodic geometry, in which blood and lymphatic capillaries are distributed uniformly throughout the tissue (see Figure 2). We modeled the lymphatic valve cycle and concluded that the interstitial deformations relax to a steady state, with a net Darcy flux per unit length of lymphatic capillary of $1.4 \times 10^{-11} \mathrm{~m}^{2} / \mathrm{s}$ and the gap length of the lymphatic valve is $0.01 \mu \mathrm{m}$ based on our physiological parameter estimates.

Validating our estimated volumetric flow rate through the initial lymphatics against experimental data is not straight forward because of the magnitude of variations in the geometrical and physical parameters of different lymphatic capillaries and in the surrounding environment. Nonetheless, we attempt to compare our results to the experimental literature on initial lymphatic uptake $[50,53,54]$ (on mice) and [24] (on rabbits). Ikomi et al [24], measured volumetric flow rates of the order of $1.1 \mathrm{~mL} / \mathrm{h}$ in the initial lymphatics of a rabbits hindleg. Using a conversion factor of 0.9 calculated in [38], which estimates the length of a single initial lymphatic channel and the total length of endothelial cell junctions in the initial lymphatics of a rabbits hind leg gives a fluid flux of $2.78 \times 10^{-10}$ $\mathrm{m}^{2} / \mathrm{s}$ per initial lymphatic channel broadly in line with our calculated value of $1.4 \times 10^{-11} \mathrm{~m}^{2} / \mathrm{s}$. Swartz et al [54] measured volumetric flow rates in the collecting lymphatics of a mouse tail skin vs the infusion pressure of the order of $10^{-8} \mathrm{~cm}^{2} / \mathrm{s}$ per $\mathrm{mmHg}$ to $10^{-9} \mathrm{~cm}^{2} / \mathrm{s}$ per $\mathrm{mmHg}$. Applying an infusion pressure of $30 \mathrm{mmHg}$ (similar to the infusion pressure in our model) gives a fluid flux of $3 \times 10^{-11} \mathrm{~m}^{2} / \mathrm{s}$ to $3 \times 10^{-12} \mathrm{~m}^{2} / \mathrm{s}$ per initial lymphatic channel (again in line with our model results). However, more recently they measured a value of the volumetric flow rate through the initial lymphatics, of the order $0.01 \mathrm{~mL} / \mathrm{min}$ [53]. They assumed 14 initial lymphatic vessels in the tail (plus the assumption of 4 valves per vessel); giving a fluid flux through the initial lymphatics of the order of $4.8 \times 10^{-11}$ $\mathrm{m}^{2} / \mathrm{s}$ per initial lymphatic channel. Reddy et al [50] measured the volumetric flow rates in the initial lymphatics of the order of $1 \times 10^{-8} \mathrm{~mL} / \mathrm{s}$ through a length of $0.25 \mathrm{~mm}$. Thus converting to fluid flux through the initial lymphatics per $\mu \mathrm{m}$ junction length gives the value of $4 \times 10^{-11} \mathrm{~m}^{2} / \mathrm{s}$ for the fluid flux. Thus we conclude that our model provides flow rates that are comparable in magnitude to those in the experimental literature. Our model also utilises more aspects of the entire lymphatic drainage (e.g. the regular deformations of the surrounding matrix to lymphatic capillaries) than previous models of the primary lymphatic 
valve system $[13,18,21,38,42]$.

It is commonly supposed that variations in lymphatic pressure play a significant role in interstitial drainage $[15,37,39,40,55,57]$. The size of such variations is typically around $100 \mathrm{~Pa}$ which compares to a mean pressure difference between blood and lymphatic capillaries of around $3000 \mathrm{~Pa}$. At the end of $\S 2$ we noted that the steady state solution to the model depends only on lymphatic pressure $P^{l}$ and blood capillary pressure $P^{b}$ through their difference $P^{b}-P^{l}$. It is thus apparent that fluctuations in the lymphatic lumen pressure provide only a small perturbation to the steady-state solution, and that the physiological state of the tissue is predominantly determined by the mean pressure difference $P^{b}-P^{l}$ (as opposed to the relatively small fluctuations in the lymphatic pressure); this is borne out by the time-dependent calculations performed in figure 12. This led us to investigate the effects of parameter variations on the steady-state in $§ 3.1$. With reference to oedema we found that physiological changes to the values of the Youngs' modulus of the tissue, the blood-lymphatic pressure difference and the blood capillary permeability only lead to small changes in the tissue swelling. This is particularly true of the Youngs' modulus $E$ (tissue swelling is almost independent of $E$ ). It is interesting to speculate on the possible advantages of a sliding valve over those of a valve operated by pressure differences between the lymphatic lumen and the surrounding tissue (as described in [20]). An evolutionary advantage of the sliding valve system over the pressure operated valve would seem to be that it responds directly to tissue swelling. It is therefore better at controlling oedema (than the pressure operated valve) even if the properties of the tissue change over time as, for example, in pregnancy, or with age. This is reflected in the fact that the extra swelling due to decreases in the tissues Youngs' modulus or increases in the blood vessels permeability are both small. The model also predicts that the degree of swelling shows some sensitivity to the endothelial cell overlap and suggests that cell overlap may be one of the traits on which selection pressure operates on to achieve an appropriate level of fluid content in the tissue.

The Darcy flux through the tissue may also have a bearing on the development of oedema since poor drainage within the secondary lymphatics (lymphoedema) can result in the system being unable to cope with large flows entering from the lymphatic capillaries. In this context we note that (I) physiological increases in Young' modulus of the tissue (see figure 8) lead to significant decreases in the flow through the interstitium; (II) physiological increases in the blood-lymphatic pressure difference (see figure 9) lead to significant increases in the flow; (III) physiological increases in the blood capillary permeability (see figure 10) lead to significant increases in the flow; and (IV) physiological increases in endothelial cell overlap (see figure 11) lead to significant decreases in the flow.

Finally, we suggest an experiment that would be able to distinguish between the Rossi 'sliding door' valve hypothesis and the more commonly postulated mechanism in which the valve valve opens in response to pressure differences between the interstitium and the lymphatic lumen. Use of a collegenase, or a matrix metalloproteinase (MMP), to degrade the extracellular matrix and lower the Young's modulus of the intersitium will result in different levels of swelling depending upon 
which of these mechanisms is in operation. If they open in response to pressure differences between the interstitium and the lymphatic lumen one would expect significantly more swelling of the tissue than if, as postulated by Rossi, they open in response to the local swelling of the interstitium around the lymphatic vessel;

in which case, as illustrated in figure 8(d), the swelling is almost independent of the Young's modulus.

\section{Acknowledgments}

CWH was funded by EPSRC PhD studentship. TR was funded by a Royal Society University Research Fellowship. 


\section{References}

[1] N. Anim-Nyame, J. Gamble, S. Sooranna, M. Johnson, \& P. Steer. Microvascular permeability is related to circulating levels of tumor necrosis factor - $\alpha$ in pre-eclampsia. Cardiovascular Res, 58: 162-169, (2003).

[2] P.J. Basser. Interstitial pressure, volume, and flow during infusion into brain tissue. Microvascular Research, 44: 143-165, (1992).

[3] D. Bates. Vascular endothelial growth factors and vascular permeability. Cardiovasc Res, 87: 202-271, (2010).

[4] L. Baxter \& R. Jain. Transport of fluid and macromolecules in tumors. ii. role of heterogeneous perfusion and lymphatics. Microvascular Research, 40: 246-263, (1990).

[5] M.A. Biot. General solutions of the equations of elasticity and consolidation for a porous material. Journal of Applied Mechanics, 78: 91-96, (1956).

[6] R. Bowen. Incompressible porous media models by use of the theory of mixtures. International Journal of Engineering Science, 18: 1129-1148, (1980).

[7] A.C. Burton. On the physical equilibrium of small blood vessels. American Physiological Society, 164: 319-329, (1951).

[8] J. Casley-Smith. The functioning and interrelationships of blood capillaries and lymphatics. Cellular and Molecular Life Sciences, 32: 1-12, (1976).

[9] S. Cho \& J.E. Atwood. Peripheral Edema. Am. J. Med., 113: 580-586, (2002).

[10] P. Danias, N. Tritos, M. Stuber, R. Botnar, K. Kissinger, \& W. Manning. Comparison of aortic elasticity determinded by cardiovascular magnetic resonance imaging in obese versus lean adults. The American Journal of Cardiology, 91: 195-199, (2003).

[11] M. Davis, M. Lane, A. Davis, D. Durtschi, D. Zawieja, M. Muthuchamy, \& A. Gasher. Modulation of Lymphatic Muscle Contractility by the Neuropeptide Substance P. The American Journal of Physiology, 295: 587-597, (2008).

[12] M. Ding, M. Dalstra, C. Danielsen, J. Tabel, I. Hvid, \& F. Linde. Age variations in the properties of human tibial trabecular bone. J. Bone Joint Surg, 79: 995-1002, (1997).

[13] J. Dixon. Lymphatic Lipid Transport: Sewer or Subway. Trends in endocrinology and metabolism, 21: 480-487, (2010). 
[14] J.B. Dixon, S.T. Greiner, A.A. Goshev, G.L. Cote, J.E. Moore Jr, \& D.C. Zawieja. Lymph flow, shear stress, and lymphocyte velocity in rat mesenteric prenodal lymphatics. Microcirculation, 13: 597-610, (2006).

[15] J. Eisenhoffer \& M. Johnston. Importance of valves and lymphangion contractions in determining pressure gradients in isolated lymphatics exposed to elevations in outflow pressure. Microvasc Res, 49: 97-110, (1995).

[16] A. Flyvbjerg, F. Dagnaes-Hansen, A. De Vriese, B. Schrijvers, R. Tilton, \& R. Rasch. Amelioration of long term renal changes in obese type 2 diabetic mice by a neutralizing vascular endothelial growth factor antibody. Diabetes, 51: 3090-3094, (2002).

[17] C. Friedman, D. Danforth, C. Herbosa-Encarnacion, L. Arbogast, B. Alak, \& D. Seifer. Follicular fluid vascular endothelial growth factor concentrations are elevated in women of advanced reproductive age undergoing ovulation induction. Fertility and Sterility, 68: 607-612, (1997).

[18] P. Galie \& R.L. Spilker. A two-dimensional computational model of lymph transport across primary lymphatic valves. Journal of Biomechanical Engineering, 131: 1297-1307, (2009).

[19] S.B. Geleff, A. Soleiman, H. Kowalski, R. Horvat, G. Amann, E. Kriehuber, K. Diem, W. Weninger, E. Tschachler, K. Alitalo, \& D. Kerjaschki. Angiosarcomas express mixed endothelial phenotypes of blood and lymphatic capillaries. American Journal of Pathology, 154: 385-394, (1999).

[20] C.W. Heppell, G Richardson, \& T Roose. A model for fluid drainage by the lymphatic system. Bull Math Biol, 75: 49-81, (2012).

[21] T. Hitchcock, \& L. Niklason. Lymphatic Tissue Engineering Progress and Prospects. Annals of the New York Academy of Sciences, 1131: 44-49, (2008)

[22] M. Holmes. Finite deformation of soft tissue: analysis of a mixture model in uni-axial compression. Journal of Biomechanical Engeering, 108: 372-381, (1986).

[23] M. Holmes. Applied Solid Mechanics Cambridge University Press, (2009).

[24] F. Ikomi, J. Hunt, G. Hanna, \& G.W. Schmid-Schönbein. Interstitial fluid, plasma protein, colliod, and leukocyte uptake into initial lymphatics. Journal of Applied Physiology, 81: 2060-2067, (1996).

[25] R.K. Jain, R.T. Tong, \& L.L. Munn. Effect of vascular normalization by antiangiogenic therapy on interstitial hypertension, peritumor edema, and lymphatic metastasis: Insights from a mathematical model. American Association for Cancer Research, 67: 2729-2735, (2007). 
[26] M. Kaczmarek, R. Subramaniam, \& S. Neff. The hydromechanics of hydrocephalus: Steady-state solutions for cylindrical geometry. Bulletin of Mathematical Biology, 59: 295-323, (1997).

[27] H. Kärkkäinen, H. Saarelainen, P. Valtonen, T. Laitinen, O. Raitakari, M. Juonala, M. Kähönen, N. Hutri-Kähönen, S. Heinonen, \& T. Laitinen. Carotid artery elasticity decreases during pregnancy - the cardiovascular risk in young finns study. BMC Pregnancy and Child Birth, 14: 98-108, (2014).

[28] T. Keaveny \& W. Hayes. A 20-year perspective on the mechanical properties of trabecular bone. J. Biomech Eng, 115: 534-542, (1993).

[29] A. Kvanter, P. Algvere, L. Berglin, \& S. Seregard. Subfoveal degeneration express vascular endothelial growth factor. Investigative Ophthalmology and Visual Science, 37: 1929-1934, (1996).

[30] L.V. Leak. Electron microscopic observations on lymphatic capillaries and the structural components of the connective tissue-lymph interface. Microvascular Research, 2: 361-391, (1970).

[31] L.V. Leak. Studies on the permeability of lymphatic capillaries. The Journal of Cell Biology, 50: 300-323, (1971).

[32] L.V. Leak \& J.F Burke. Ultrastructural studies on the lymphatic anchoring filmants. The Journal of Cell Biology, 36: 129-149, (1968).

[33] J.S. Lee. Tissue fluid pressure, lymph pressure, and fluid transport in rat intestinal villi. Microvascular Research, 31: 170-183, (2004).

[34] J.R. Levick. Flow through interstitium and other fibrous matrices. Journal of Experimental Phsyiology, 72: 409-439, (1987).

[35] M. Macedo, D. Luminoso, M. Savvidou, C. McEniery, \& K. Nicolaides. Pregnancy, hypertension, and preeclampsia. Hypertension, 51: 1047-1051, (2008).

[36] K.N. Margaris \& R.A. Black. Modelling the lymphatic system: challenges and opportunities. Journal of The Royal Society Interface, 9: 601-612, (2012).

[37] A. Mattei, F. Fuechsel, N. Dhar, S. Warnike, G. Thalmann, T. Krause, \& U. Studer. The Template of the Primary Lymphatic Landing Sites of the Prostate should be Revisted: Results of a Multimodality Mapping Study. European Urology, 53: 118-125, (2008).

[38] E. Mendoza \& G.W. Schmid-Schönbein. A model for mechanics of primary lymphatic valves. Journal of Biomechanical Engineering, 125: 407-414, (2003).

[39] A. Moriondo, F. Boschetti, F. Bianchin, S. Lattanzio, C. Marcozzi, \& D. Negrini. Tissue contribution to the mechanical features of diaphragmatic initial lymphatics. The Journal of Physiology, 588: 3957-3969, (2010). 
[40] M. Nipper \& J. Dixon. Engineering the Lymphatic System. Cardiovascular engineering and technology, 2: 296-308, (2011).

[41] J. Pusenjak \& D. Miklavcic. Modeling of interstitial fluid pressure in solid tumor. Simulation Practice and Theory, 8: 17-24, (2000).

[42] N.P. Reddy \& K. Patel. A mathematical model of flow through the terminal lymphatics. Medical Engineering and Physics, 17: 134-140, (1994).

[43] T. Roose, \& A. Fowler. Network Development in Biological gels: roles in Lymphatic Vessel Development. Bulletin of Mathematical Biology, 70: 17721789, (2008)

[44] T. Roose, \& M. Swartz. Multiscale Modeling of Lymphatic drainage from Tissues using Homogenization Theory. Journal of Biomechanics, 45: $107-$ 115, (2012)

[45] T. Roose, \& G. Tabor. Multiscale Modelling of Lymphatic Drainage. Journal of Biomechanics, 14: 149-176, (2013)

[46] A. Rossi, E. Weber, G. Sacchi, D. Maestrini, F. Di Cintio, \& R. Gerli. Mechanotransduction in lymphatic endothelial cells. Lymphology, 40: 102$113,(2007)$.

[47] M. Sarntinoranont, F. Rooney, \& M. Ferrari. Interstitial stress and fluid pressure within a growing tumor. Biomedical Engineering Society, 31: $327-$ $335,(2003)$.

[48] B. Sauter, D. Foedinger, B. Sterniczky, K. Wolff, \& K. Rappersberger. Immunoelectron microscopic characterization of human dermal lymphatic microvascular endothelial cells: … Journal of Histochemistry and Cytochemistry, 46: 165-176, (1998).

[49] M. Sherratt. Tissue elasticity and the ageing elastic fibre. Age, 31: 305-325, (2009).

[50] S.T Reddy, D.A Berk, R.K Jain, \& M.A. Swartz A sensitive in vivo model for quantifying interstitial convective transport of injected macromolecules and nanoparticles. Journal of Applied Physics, 101: 1162-1169, (2006).

[51] N. Staub. Pulmonary edema due to increased microvascular permeability to fluid and protein. Circulation Res, 43: 143-151, (1978).

[52] M.A. Swartz. The physiology of the lymphatic system. Advanced Drug Delivery Reviews, 50: 3-20, (2001).

[53] M.A Swartz \& K.C. Boardman. The role of interstitial stress in lymphatic function and lymphangiogenesis. Annals of the New York Academy of Sciences, 979: 197-210, (2002). 
[54] M.A. Swartz, A. Kaipainen, P.A. Netti, C. Brekken, Y. Boucher, A.J. Grodzinsky, \& R.K. Jain. Mechanics of interstitial-lymphatic fluid transport: theoretical foundation and experimental validation. Journal of Biomechanics, 32: 1297-1307, (1999).

[55] J. Unthank \& H. Bohlen. Lymphatic pathways and role of valves in lymph propulsion from small intestine. Am. J. Physiol., 254: 389-398, (1988).

[56] R. Wilaman, R. Mackey, A. Bostom, T. Thompson, \& K. Sulton-Tyrrell. Measures of obesity are associated with vascular stiffness in young and older adults. Hypertension, 42: 408-473, (2003).

[57] B. Zweifach \& J. Prather. Micromanipulation of pressure in terminal lymphatics in the mesentery. Am. J. Physiol., 228: 1326-1335, (1975). 


\section{A Numerical solution of the Model using Com- sol}

Here we describe how we use the finite element package Comsol Multiphysics (CM) to solve the model formulated in section 2. At its most basic this comprises of comparing the poroelastiticity equations defined in CM to equations (2.7) and (2.11) and interpreting the boundary conditions (2.18)-(2.31) in a form appropriate for CM and then solving for the solid matrix deformations and the interstitial fluid flow.

\section{A.1 The Poroelastic Equations}

$\mathrm{CM}$ has a poroelastic module that can be used to find numerical solutions to Biot's equations. This couples together a Darcy's Law interface (for the flow) to a Solid Mechanics Interface (for the mechanical deformations) which we describe below.

\section{A.1.1 Darcy's Law Interface}

This is used to determine fluid motion through a porous medium. Darcy's law states that the fluid flux $\left(\mathbf{Q}^{f}\right)$ is linearly related to the pressure gradient in the medium $(\nabla P)$ via the relation

$$
\mathrm{Q}^{f}=-\frac{\kappa}{\mu} \nabla P
$$

where $\mu$ is fluid viscosity and $\kappa$ the permeability of the porous medium (for consistency with $\S 2 K=\kappa / \mu)$. This is solved together with the fluid continuity equation, which is provided by the Poroelasticity Interface

$$
\nabla \cdot\left(\mathbf{v}^{f}\right)=-\alpha_{b} \frac{\partial}{\partial t}(\nabla \cdot \mathbf{u})
$$

where for consistency with $\S 2$ we set the Biot-Willis coefficient $\alpha_{b}=1$.

\section{A.1.2 Solid Mechanics Interface}

Following the small displacement assumption, the normal strain components and the shear strain components, $\epsilon_{i j}$, are given from the deformations, $u_{i}$, via

$$
\epsilon_{i j}=\frac{1}{2}\left(\frac{\partial u_{i}}{\partial x_{j}}+\frac{\partial u_{j}}{\partial x_{i}}\right) .
$$

and, since we assume plane strain $\boldsymbol{u}=\left(u_{1}\left(x_{1}, x_{2}\right), u_{2}\left(x_{1}, x_{2}\right), 0\right)$, it follows that $\epsilon_{13}=\epsilon_{23}=\epsilon_{33}=0$.

For a poroelastic material the stress tensor $\left(\sigma_{i j}\right)$ is related to the the strain tensor by the standard linear constitutive relation $\sigma_{i j}=D_{i j k l} \epsilon_{k l}-P \alpha_{b} \delta_{i j}$ where 
the term $-P \alpha_{b} \delta_{i j}$ is added to account for the pore pressure (here $D_{i j k l}$ is entered into the Solid Mechanics Interface). For isotropic materials this can be reduced (see e.g. [23]) to the form

$$
\sigma_{i j}=\lambda e_{k k} \delta_{i j}+2 \mu e_{i j}-P \alpha_{b} \delta_{i j}, \text { where } \lambda=\frac{E \nu}{(1+\nu)(1-2 \nu)}, \mu=\frac{E}{2(1+\nu)} .
$$

where $E$ is Young's modulus and $\nu$ is the Poisson's ratio. Under the assumptions of plane strain $\sigma_{13}=\sigma_{31}=0$ and the force balance equations (that are entered into the structural mechanics module) are

$$
\begin{aligned}
& \frac{\partial \sigma_{11}}{\partial x_{1}}+\frac{\partial \sigma_{12}}{\partial x_{2}}=0, \\
& \frac{\partial \sigma_{21}}{\partial x_{1}}+\frac{\partial \sigma_{22}}{\partial x_{2}}=0
\end{aligned}
$$

The stresses playing a role in the force balance equation are related to the non-zero strain tensor compents via

$$
\left[\begin{array}{l}
\sigma_{11} \\
\sigma_{22} \\
\sigma_{12}
\end{array}\right]=\frac{E}{(1+\nu)(1-2 \nu)}\left[\begin{array}{ccc}
1-\nu & \nu & 0 \\
\nu & 1-\nu & 0 \\
0 & 0 & 1-2 \nu
\end{array}\right]\left[\begin{array}{c}
\epsilon_{11} \\
\epsilon_{22} \\
\epsilon_{12}
\end{array}\right]-\alpha_{b} P\left[\begin{array}{l}
1 \\
1 \\
0
\end{array}\right] \text {. }
$$

The governing equations are (A.1)-(A.3) and (A.5)-(A.7), with $\alpha_{b}=1$, are identical to those in $\S 2$ (i.e. $(2.7)$ and $(2.11)$ ).

\section{A.1.3 Boundary Conditions}

Here we convert our boundary conditions (on the sections of boundary illustrated in figure 3) to a form usable by CM.

\section{The lymphatic capillary wall $\partial \Omega_{4}$}

The lymphatic capillary wall $\left(\partial \Omega_{4}\right)$ is split into two parts, which are denoted by $\partial \Omega_{4 a}$ and $\partial \Omega_{4 b}$, see Figure 3 . Here the boundary $\partial \Omega_{4 a}$ corresponds to the open section of the lymphatic valve (through which interstitial fluid flows) while $\partial \Omega_{4 b}$ represents the endothelial cell wall (through which there is no flow). We assume that the open section of the lymphatic valve is located half way along the capillary boundary, as illustrated in Figure 3.

We determine the posiitions of $\partial \Omega_{4 a}$ and $\partial \Omega_{4 b}$ by introducing two new variables

$$
\begin{aligned}
\theta_{s} & =\frac{\pi C_{\text {crit }}}{4 C(t)} \\
\theta_{f} & =\frac{\pi\left(2 C(t)-C_{\text {crit }}\right)}{4 C(t)} .
\end{aligned}
$$

Here $\theta_{s}$ and $\theta_{f}$ are the angles from the horizontal to the start of $\partial \Omega_{4 a}$ and the end of $\partial \Omega_{4 a}$ respectively. Thus $\partial \Omega_{4 a}=\left\{\theta \mid \theta \in\left[\theta_{s}, \theta_{f}\right]\right\}$ and $\partial \Omega_{4 b}=\left\{\theta \mid \theta \in\left[0, \theta_{s}\right)\right\} \cup\{\theta \mid \theta \in$ $\left.\left(\theta_{f}, \pi / 2\right)\right\}$ where $\theta$ is the angle from the horizontal. 
Using these angles in CM allows us to use a single flow boundary condition on $\partial \Omega_{4}$ and still incorporate the valve mechanics. We determine $C(t)$ by evaluating the boundary integral $C(t)=\int_{\partial \Omega_{4}(t)} d S$. The assumption that the lymphatic valve is closed when $C(t)<C_{\text {crit }}$, and open when $C(t)>C_{\text {crit }}$ implies there is no flow into the lumen if $C(t)<C_{\text {crit }}$ and that fluid flows into the lumen only through $\partial \Omega_{4 a}$ when $C(t)>C_{\text {crit }}$. The boundary condition is implemented in CM by the command

$$
\nabla P \cdot \mathbf{n}=\left(\nabla P \cdot \mathbf{n}+P-P^{l}\right) \times \operatorname{if}\left(\theta_{s}<\theta<\theta_{f}, 1,0\right) .
$$

This implies that

$$
\begin{aligned}
P & =P^{l} \quad \text { on } \quad \mathbf{x} \in \partial \Omega_{4 a}\left(\text { i.e. } \theta_{s}<\theta<\theta_{f}\right) \\
\nabla P \cdot \mathbf{n} & =0 \text { on } \mathbf{x} \in \partial \Omega_{4 b}\left(\text { i.e. } 0<\theta<\theta_{s} \text { and } \pi / 2>\theta>\theta_{f}\right)
\end{aligned}
$$

where $P^{l}$ is the fluid pressure of the lymphatic lumen. It is thus equivalent to (2.18)-(2.19), the conditions derived in $\S 2$.

\section{The blood capillary wall, boundary $\partial \Omega_{1}$}

The blood capillary wall is permeable to fluid but provides a resistance to it. We use the boundary condition Pervious Layer in CM to model the flow through this wall. This describes a mass flux through a semi-pervious layer connected to an external fluid source at given pressure $\left(P^{b}\right)$ and is equivalent to

$$
\frac{\kappa}{\mu} \nabla P \cdot \mathbf{n}=L_{p}\left(P-P^{b}\right) \quad \text { on } \quad \mathbf{x} \in \partial \Omega_{1},
$$

and so, where $\kappa / \mu=K$, is identical to (2.20).

Remaining edges of the periodic tile, boundaries $\partial \Omega_{2}, \partial \Omega_{3}, \partial \Omega_{5}$ and $\partial \Omega_{6}$

The conditions (2.22), namely

$$
\nabla P \cdot \mathbf{n}=0 \quad \text { on } \quad \mathbf{x} \in \partial \Omega_{2} \cup \partial \Omega_{3} \cup \partial \Omega_{5} \cup \partial \Omega_{6},
$$

are represented by the 'No Flow' feature in CM.

\section{Solid Boundary Conditions}

Since the periodic tile must remains square as it expands and contracts its edges have to remain horizontal $\left(\partial \Omega_{2}\right.$ and $\left.\partial \Omega_{5}\right)$ and vertical $\left(\partial \Omega_{3}\right.$ and $\left.\partial \Omega_{6}\right)$. And without loss of generality we impose zero normal tissue displacement on boundaries $\partial \Omega_{2}$ and $\partial \Omega_{6}$ but allow them to deform freely in the tangential direction; the latter is equivalent to imposing zero tangential shear stress along the boundaries. These conditions are represented by the Roller condition in CM, that is

$$
\begin{aligned}
& u_{2}=0 \text { and } \sigma_{21}=0 \quad \text { on } \quad \mathbf{x} \in \partial \Omega_{2} \text {, } \\
& u_{1}=0 \text { and } \sigma_{12}=0 \quad \text { on } \quad \mathbf{x} \in \partial \Omega_{6} \text {. }
\end{aligned}
$$


The edges $\partial \Omega_{3}$ and $\partial \Omega_{5}$ of the periodic tile deform freely in tangential direction (equivalent to tangential shear stress). However both of these edges are displaced normally by a distance $u_{c}(t)$, which we shall calculate. The Prescribed Displacement feature in CM imposes a condition in which the position of the boundary of the solid matrix is prescribed in one direction and free to deform in the other direction, and in our case is used to impose the conditions

$$
\begin{aligned}
& u_{1}=u_{c}(t) \quad \text { and } \quad \sigma_{12}=0 \quad \text { on } \quad \mathbf{x} \in \partial \Omega_{3}, \\
& u_{2}=u_{c}(t) \quad \text { and } \quad \sigma_{21}=0 \quad \text { on } \quad \mathbf{x} \in \partial \Omega_{5},
\end{aligned}
$$

where $u_{c}(t)$ is calculated by

$$
u_{c}(t)=\frac{1}{2 L(t)}\left(\int_{\partial \Omega_{1} \cup \partial \Omega_{4}}\left[\int K \nabla P \cdot \mathbf{n} d t\right] d S-\int_{\partial \Omega_{1} \cup \partial \Omega_{4}} \mathbf{u} \cdot \mathbf{n} d S\right) .
$$

Here $L(t)$ is the width of the periodic tile, which is a square, minus the radius of the lymphatic capillary.

The first integral term on the right hand side of (A.19) cannot be calculated directly in $\mathrm{CM}$ because it is a time dependent integral and the inbuilt time integrals in CM (timeint and timeavg) are only available during the results evaluation. In order to overcome this difificulty we introduce the new time dependent variable, $T_{1}(t)$, defined as the solution to

$$
\frac{d T_{1}}{d t}=\int_{\partial \Omega_{1} \cup \partial \Omega_{4}} K \nabla P \cdot \mathbf{n} d S \text { and } T_{1}(0)=0,
$$

so that

$$
T_{1}(t)=\int_{\partial \Omega_{1} \cup \partial \Omega_{4}}\left[\int K \nabla P \cdot \mathbf{n} d t\right] d S .
$$

It follows from (2.19) and (2.20) that

$$
\frac{d T_{1}}{d t}=\int_{\partial \Omega_{1}} L_{p}\left(P-P^{b}\right) d S+\int_{\partial \Omega_{4 a}} K \nabla P \cdot \mathbf{n} d S
$$

However the tangential derivative of $P$ on $\partial \Omega_{4 a}$ is zero $\left(i . e .\left.\nabla P \cdot \boldsymbol{t}\right|_{\partial \Omega_{4 a}}=0\right.$ ), since $P=P^{l}$ on this section of boundary. It follows that $\nabla P \cdot \mathbf{n}=|\nabla P|$ (here we expect $\nabla P \cdot \boldsymbol{n}>0$ since the pressure acts outward on the boundary). We can thus rewrite (A.21) as

$$
\frac{d T_{1}}{d t}=\int_{\partial \Omega_{1}} L_{p}\left(P-P^{b}\right) d S+\int_{\partial \Omega_{4 a}} K\left(\left(\frac{\partial P}{\partial x_{1}}\right)^{2}+\left(\frac{\partial P}{\partial x_{2}}\right)^{2}\right)^{1 / 2} d S .
$$

Since both integrals can be integrated straightforwardly using CM the ODE for $T_{1}(t)$ can be solved by CM. This allows $u_{c}(t)$ to be determined, since the second integral term on the right hand side of (A.19) is readily evaluated using CM. 
Blood and lymphatic capillary, boundaries $\partial \Omega_{1}$ and $\partial \Omega_{4}$

Boundary conditions (2.30)-(2.31) can be implemented striaghtforwardly in CM using the Boundary Load feature which describes a boundary that experiences zero tangential stresses but on which the normal stresses are balanced by an external pressure. 